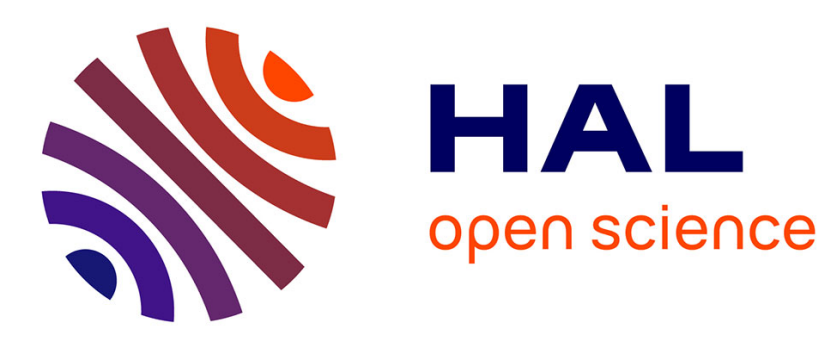

\title{
Identification of random shapes from images through polynomial chaos expansion of random level-set functions
}

\author{
G. Stefanou, Anthony Nouy, Alexandre Clement
}

\section{- To cite this version:}

G. Stefanou, Anthony Nouy, Alexandre Clement. Identification of random shapes from images through polynomial chaos expansion of random level-set functions. International Journal for Numerical Methods in Engineering, 2009, 79 (2), pp.127-155. 10.1002/nme.2546 . hal-00366640

\section{HAL Id: hal-00366640 \\ https://hal.science/hal-00366640}

Submitted on 9 Mar 2009

HAL is a multi-disciplinary open access archive for the deposit and dissemination of scientific research documents, whether they are published or not. The documents may come from teaching and research institutions in France or abroad, or from public or private research centers.
L'archive ouverte pluridisciplinaire HAL, est destinée au dépôt et à la diffusion de documents scientifiques de niveau recherche, publiés ou non, émanant des établissements d'enseignement et de recherche français ou étrangers, des laboratoires publics ou privés. 


\title{
Identification of random shapes from images through polynomial chaos expansion of random level-set functions
}

\author{
G. STEFANOU, A. NOUY*†, A. CLEMENT \\ Research Institute in Civil Engineering and Mechanics (GeM), \\ University of Nantes, Ecole Centrale Nantes, UMR CNRS 6183 \\ 2 rue de la Houssinière, 44322 Nantes, France
}

\begin{abstract}
SUMMARY
In this paper, an efficient method is proposed for the identification of random shapes in a form suitable for numerical simulation within the eXtended Stochastic Finite Element Method (X-SFEM). The method starts from a collection of images representing different outcomes of the random shape to identify. The key-point of the method is to represent the random geometry in an implicit manner using the level-set technique. In this context, the problem of random geometry identification is equivalent to the identification of a random level-set function, which is a random field. This random field is represented on a polynomial chaos basis and various efficient numerical strategies are proposed in order to identify the coefficients of its polynomial chaos decomposition. The performance of these strategies is evaluated through some "manufactured" problems and useful conclusions are provided. The propagation of geometrical uncertainties in structural analysis using the X-SFEM is finally examined.
\end{abstract}

KEY WORDS: Random geometry; Level-set method; Probabilistic identification; Polynomial chaos; Maximum likelihood; Extended stochastic finite element method.

\section{Introduction}

The considerable influence of inherent uncertainties on the behavior of physical systems has led the scientific community to recognize the importance of a stochastic approach. Issues related to uncertainty quantification and its influence on the reliability of the computational models, are continuously gaining in significance. In the last decades, an increasing interest has been devoted to the development of numerical techniques such as stochastic finite elements [1, 2], in order to assess the impact of randomness on the response of physical systems governed by stochastic partial differential equations. Uncertainties in the differential operator and source

\footnotetext{
${ }^{*}$ Correspondence to: Anthony Nouy : Research Institute in Civil Engineering and Mechanics (GeM), 2 rue de la Houssinière, 44322 Nantes, France

†E-mail: anthony.nouy@univ-nantes.fr

Grant sponsor: French National Research Agency; grant number: ANR-06-JCJC-0064
} 
terms are quite well mastered in the framework of these techniques. However, there are only a few available numerical strategies to deal with uncertainties in the geometry although it is of great interest in various applications. Some representative examples of mechanical problems with random geometry are the following: holes with random shapes in structural components, shells with cutouts where the size and shape of the cutout is random due to imperfections in the manufacturing procedure, random distribution of inclusions within heterogeneous materials (e.g. fiber reinforced composites, concrete). The effect of randomness in the geometry on the response of these systems may be important. It is thus imperative to be able to correctly identify the randomness in the geometry of physical systems.

The identification of a probabilistic model is a very critical point. It usually requires many samples and robust identification techniques of random variables or fields. Experimental campaigns or in-site measurements are often very expensive, a fact that drastically limits the number of available samples and thus the quality of identification. Different experimental means can be used to identify random shapes: thermal imaging, optical and X-ray tomography, image processing. Among these techniques, shape recovery from simple images ("pictures" of the shape) has the following advantages: it is non-intrusive, it allows obtaining many samples at a very low cost and it can be relatively precise. This identification procedure leads to a characterization of the random geometry in a form that can be directly used for the numerical simulation of the physical problem. For the computation of the response, one generally has to solve partial differential equations defined on random domains. The number of numerical strategies proposed for this kind of problems is limited [3, 4]. Recently, the eXtended Stochastic Finite Element Method (X-SFEM) [5, 6] has been proposed. This approach, which is an extension to the stochastic framework of the X-FEM method [7, 8, 9, 10, 11], relies on the implicit representation of complex geometries using random level-set functions and on the use of a Galerkin approximation at both stochastic and deterministic levels.

The methodology proposed in this paper is an efficient identification procedure of random geometry in a form suitable for numerical simulation within the X-SFEM. The method starts from a collection of images, representing different outcomes of the random shape to identify. The key-point is to represent the random geometry in an implicit manner using the levelset technique [12]. This technique consists in representing the boundary of a shape with a level-set function, which is the signed distance function to the boundary. This technique is well known and mastered in the context of shape recovery from images [12]. In our case, the random geometry will be characterized by a random level-set function, one outcome of which represents an outcome of the random boundary of the shape to be recovered. The problem of random geometry identification is thus equivalent to the identification of a random level-set function, which is a random field. Some general techniques for the identification of random fields have been proposed in $[13,14]$. These techniques consist in the representation of the random field on a polynomial chaos basis. Here, this idea is adopted and the identification of a polynomial chaos representation of the random level-set is performed. More precisely, some efficient numerical strategies are proposed in order to identify the coefficients of the decomposition.

The remainder of the paper is organized as follows: in section 2, we describe the principle of shape recovery from image with the level-set technique. It allows the construction of samples of the random level-set from a collection of images, associated with samples of the random shapes. In section 3, we introduce an empirical Karhunen-Loève decomposition of the samples, which allows to represent the samples of the level-set on a reduced basis of deterministic modes. 
It thus transforms the problem into the probabilistic identification of a few random variables, which are the components of the random level-set on this reduced basis of modes. In sections 4 and 5, some methodologies are presented in order to identify a polynomial chaos representation of random variables. In section 4 , we deal with the general case of mutually dependent random variables, while in section 5 , a hypothesis of independence of random variables is introduced. Section 6 will illustrate the methodologies on "manufactured" problems. In section 7, we will focus on the propagation of geometrical uncertainties in structural analysis with the X-SFEM.

\section{Shape recovery with the level-set technique}

The problem of shape recovery from an image is now a well-known and mastered problem within the context of level-set techniques [12]. Here, we briefly recall the basis of this method. We suppose that we have a contrasted image, defined by a mapping $I: \boldsymbol{x} \in D \rightarrow \mathbb{R}$, whose value $I(\boldsymbol{x})$ represents the grayness intensity at location $\boldsymbol{x} \in D$ (where $D \subset \mathbb{R}^{2}$ or even $D \subset \mathbb{R}^{3}$ ). The aim is to detect the boundary of the underlying shape. This boundary is in fact located in the region where the intensity has the highest gradients. The aim of shape recovery consists in building a level-set function $\phi(\boldsymbol{x})$ whose iso-zero is located in this region with high intensity gradients. The basic idea consists in propagating a front, represented by this iso-zero of a time-dependent level-set $\phi(\boldsymbol{x}, t)$, which will "lock" on the desired boundary. The well-known equation of motion of a level-set $\phi(\boldsymbol{x}, t)$ writes:

$$
\begin{aligned}
& \partial_{t} \phi(\boldsymbol{x}, t)+F(\boldsymbol{x}, t)\|\nabla \phi(\boldsymbol{x}, t)\|=0 \\
& \phi(\boldsymbol{x}, 0)=\phi_{0}(\boldsymbol{x})
\end{aligned}
$$

where $F$ is the speed of the front in the outward normal direction (from negative to positive values of $\phi$ ). In order to make the iso-zero lock in high intensity gradients zones, the speed has to vanish in these zones. A classical choice for $F$ (see [12]) consists in taking:

$$
F=(1-\epsilon \kappa) \frac{1}{1+c\left\|\nabla\left(G_{\sigma} * I\right)\right\|}
$$

where $\kappa$ is the curvature of the front $\left(\kappa=\nabla \cdot\left(\frac{\nabla \phi}{\|\nabla \phi\|}\right)\right), \epsilon>0$ a small parameter, $I$ the mapping of grayness intensity and $G_{\sigma}$ a Gaussian smoothing filter with characteristic width $\sigma . \nabla\left(G_{\sigma} * I\right)$ represents the gradient of the image convolved with the filter. The curvature term is a classical regularization term, leading to a smooth front. Parameter $c$ allows imposing an arbitrary small value of the speed in high intensity gradients zones.

A basic choice for the initial level-set $\phi_{0}$ consists in a small circular front in the interior of the boundary to be recovered. Many algorithms have been proposed in order to solve equation of motion (1) (see [12]). After discretization and resolution, this leads to a discretized level-set $\phi \in \mathbb{R}^{N}$.

Remark 1. The initial front can also consist in the union of small circular fronts centered at different arbitrary points. The level-set representation allowing changes in the topology of the front, these initial fronts can naturally merge into a simpler front, eventually simply connected.

Illustration We consider the case of Figure 1, showing a filtered image and its gradient. Here, we have used the Matlab Toolbox for Level-set Methods [15] for solving equation (1). This equation is solved with a finite-difference scheme on a regular spatial grid of size $60 \times 60$. 


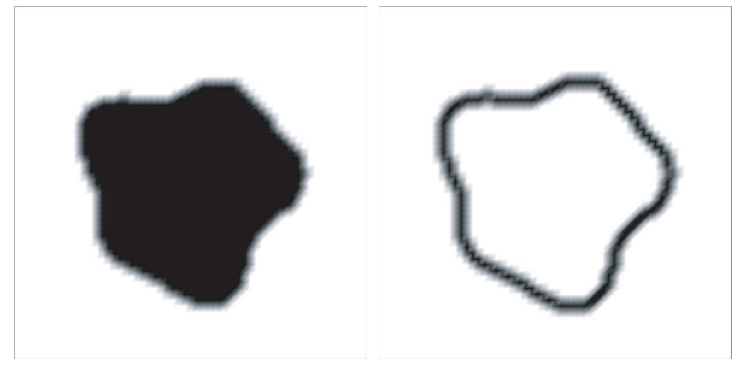
(a) $G_{\sigma} * I$
(b) $\left\|\nabla\left(G_{\sigma} * I\right)\right\|$

Figure 1. (a) Mapping of grayness intensity $I$ convolved with a Gaussian filter $G_{\sigma}$ and (b) gradient of the filtered image

Figure 2 shows the iso-zero of levet-set $\phi(x, t)$ at different time steps during the resolution. We observe that the asscociated front "locks" on the high gradient zone.

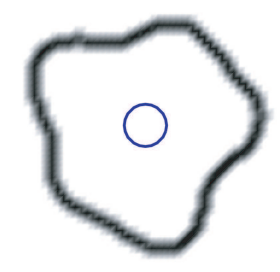

(a) $t=0$.

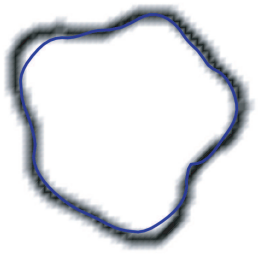

(d) $t=0.42$

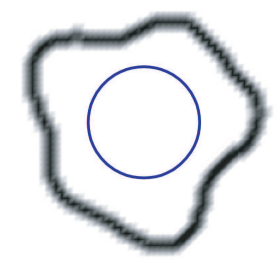

(b) $t=0.14$

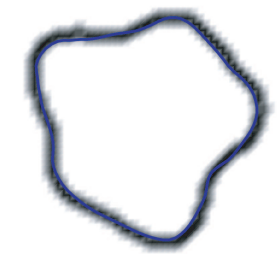

(e) $t=0.71$

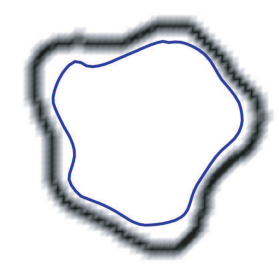

(c) $t=0.28$

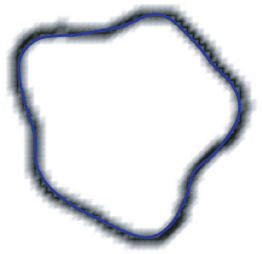

(f) $t=1$

Figure 2. Iso-zero of level-set $\phi(\boldsymbol{x}, t)$ at different time instants $t$

3. Reduction of information through Karhunen-Loève decomposition

In our problem, the random shape is characterized by a discretized random level-set, represented by a random vector $\phi: \Theta \rightarrow \mathbb{R}^{N}$ defined on an abstract probability space $(\Theta, \mathcal{B}, P)$. 
By applying $Q$ times the above shape recovery technique to a set of sample images $\left\{I^{(k)}\right\}_{k=1}^{Q}$, we obtain the corresponding sample discretized level-sets $\left\{\phi^{(k)}\right\}_{k=1}^{Q}$, with $\phi^{(k)} \in \mathbb{R}^{N}$.

The probabilistic identification of the random discretized level-set $\phi$ from samples $\left\{\phi^{(k)}\right\}$ is infeasible in practice, due to the high dimensionality of the underlying probabilistic inverse problem. A reduction of information is thus unavoidable. This reduction of information simply consists in expressing the level-set in terms of a small set of random variables $\left(X_{1}, \ldots, X_{n}\right):=\mathbf{X}$. The problem of the identification of the random level-set will be then transformed into the problem of the identification of a smaller random vector $\mathbf{X}(\theta)$ from a collection of samples $\left\{\mathbf{X}^{(k)}\right\}_{k=1}^{Q}$.

In the general case where the shape is not known a priori, we propose to perform a reduction of information through discrete empirical Karhunen-Loève (K-L) decomposition [16] of levelset samples. This kind of reduction is also known as Principal Component Analysis [17] or Singular Value Decomposition and has been used in various domains of application such as functional data analysis [18, 19], image analysis [20], dynamical model reduction [21, 22], etc. By this decomposition, we try to extract from samples a few representative spatial modes on which the level-set samples can be decomposed. Then, components of the level-set samples on this reduced basis of modes are the samples of random variables to be identified.

\subsection{Empirical Karhunen-Loève decomposition}

Let us denote by $\mu_{\phi}$ the empirical mean of level-set samples $\phi^{(k)}$ and by $\widetilde{\phi}^{(k)}=\phi^{(k)}-\boldsymbol{\mu}_{\phi}$ the centered samples. The unbiased empirical covariance matrix of samples writes:

$$
\mathbf{C}_{\phi}=(Q-1)^{-1} \sum_{k=1}^{Q} \widetilde{\phi}^{(k)} \widetilde{\phi}^{(k) T}
$$

We denote by $\left\{\left(s_{i}, \mathbf{U}_{i}\right)\right\}_{i=1}^{N}$ the eigenpairs (eigenvalues, eigenvectors) of $\mathbf{C}_{\boldsymbol{\phi}}$. Vectors $\mathbf{U}_{i}$ form an orthonormal basis of $\mathbb{R}^{N}$. Then, level-set samples can be decomposed in the following way:

$$
\phi^{(k)}=\boldsymbol{\mu}_{\boldsymbol{\phi}}+\sum_{i=1}^{N} \sqrt{s_{i}} \mathbf{U}_{i} X_{i}^{(k)}, \quad X_{i}^{(k)}=\frac{1}{\sqrt{s_{i}}} \mathbf{U}_{i}^{T} \boldsymbol{\phi}^{(k)}
$$

where the $\left\{X_{i}^{(k)}\right\}_{i=1}^{N}$ appear as the components of $\widetilde{\phi}^{(k)}$ on the basis of modes $\left\{\sqrt{s_{i}} U_{i}\right\}_{i=1}^{N}$.

\subsection{Reduction of information by truncation}

Modes are sorted by decreasing eigenvalues, i.e. $s_{1} \geqslant \ldots \geqslant s_{N}$. We can then only retain the $m$ most significant modes:

$$
\phi^{(k)} \approx \hat{\phi}^{(k)}=\mu_{\boldsymbol{\phi}}+\sum_{i=1}^{m} \sqrt{s_{i}} \mathbf{U}_{i} X_{i}^{(k)}
$$

We classically show that the approximation error verifies:

$$
\frac{1}{Q-1} \sum_{k=1}^{Q}\left\|\boldsymbol{\phi}^{(k)}-\hat{\boldsymbol{\phi}}^{(k)}\right\|^{2}=\sum_{i=m+1}^{N} s_{i}
$$


where $\|\cdot\|$ denotes the classical 2-norm in $\mathbb{R}^{N}$. The number of modes $m$ to be kept can then be chosen as the smallest integer $m$ such that

$$
\sum_{i=m+1}^{N} s_{i} \leqslant \epsilon_{K L}^{2} \sum_{i=1}^{N} s_{i}
$$

where $\epsilon_{K L}$ is a tolerance which is fixed a priori and allows controlling the reduction error. Finally, samples of the level-set are approximated by:

$$
\phi^{(k)} \approx \boldsymbol{\mu}_{\phi}+\sum_{i=1}^{m} \widetilde{\mathbf{U}}_{i} X_{i}^{(k)}, \quad \widetilde{\mathbf{U}}_{i}=\sqrt{s_{i}} \mathbf{U}_{i}
$$

The $\left\{X_{i}^{(k)}\right\}_{k=1}^{Q}$ appear as samples of a random variable $X_{i}(\theta)$ representing the component of the random level-set on mode $\widetilde{\mathbf{U}}_{i}$. One then has to identify the random vector $\mathbf{X}(\theta)=$ $\left(X_{1}(\theta) \ldots X_{m}(\theta)\right)^{T}$, knowing its $Q$ samples $\mathbf{X}^{(k)}=\left(X_{1}^{(k)} \ldots X_{m}^{(k)}\right)^{T}$. With the chosen definition of $\mathbf{X}^{(k)}$, empirical mean and unbiased covariance matrix of samples satisfy:

$$
Q^{-1} \sum_{k=1}^{Q} \mathbf{X}^{(k)}=0, \quad(Q-1)^{-1} \sum_{k=1}^{Q} \mathbf{X}^{(k)} \mathbf{X}^{(k)^{T}}=\mathbf{I}_{n} .
$$

The $X_{i}$ to be identified can then be considered as centered normalized uncorrelated random variables.

\section{Polynomial chaos representation of random variables}

The problem is now to identify a random vector $\mathbf{X}: \Theta \rightarrow \mathbb{R}^{m}$, defined on an abstract probability space $(\Theta, \mathcal{B}, P)$, knowing a set of independent samples $\left\{\mathbf{X}^{(k)}\right\}_{k=1}^{Q}$. Random variables $X_{i}(\theta)$ are supposed to be centered normalized uncorrelated random variables. In this section, we don't make any further assumption and identify a random vector with arbitrary probability law. For this purpose and following [13], we use a polynomial chaos (PC) representation of $\mathbf{X}$, identified with a maximum likelihood principle. This leads to the resolution of an optimization problem on a Stiefel manifold. A numerical strategy is proposed for solving the resulting optimization problem. Section 5 will introduce a great simplification, which can be often made in practice, by assuming independence of random variables.

\subsection{Polynomial chaos decomposition}

We consider that $\mathbf{X}$ is a second order random vector and that there exists a non-linear mapping g: $\mathbb{R}^{m} \rightarrow \mathbb{R}^{m}$ such that $\mathbf{X}=\mathbf{g}(\boldsymbol{\xi})$, where $\boldsymbol{\xi}=\left(\xi_{1}, \ldots, \xi_{m}\right)$ is a $m$-dimensional random vector of independent random variables with known probability law $P_{\boldsymbol{\xi}}$ and support $\boldsymbol{\Xi} \subset \mathbb{R}^{m}$. We denote by $\left(\boldsymbol{\Xi}, \mathcal{B}_{\boldsymbol{\Xi}}, P_{\boldsymbol{\xi}}\right)$ the $m$-dimensional probability space defined by random vector $\boldsymbol{\xi}$. Random vector $\mathbf{X}$ then admits a generalized chaos representation [23, 24] writing:

$$
\mathbf{X}(\theta)=\sum_{\boldsymbol{\alpha} \in \mathcal{J}_{m}} \mathbf{X}_{\boldsymbol{\alpha}} H_{\boldsymbol{\alpha}}(\boldsymbol{\xi}(\theta))
$$


where $\left\{H_{\boldsymbol{\alpha}}\right\}_{\boldsymbol{\alpha} \in \mathcal{J}_{m}}$ is a Hilbertian basis of $L^{2}\left(\boldsymbol{\Xi}, d P_{\boldsymbol{\xi}}\right)$, endowed with its natural inner product. Coefficients $\mathbf{X}_{\boldsymbol{\alpha}}$ of the decomposition are then simply defined as the $L^{2}$-projection of $\mathbf{g}$ on the $H_{\boldsymbol{\alpha}}: \forall \boldsymbol{\alpha} \in \mathcal{J}_{m}$,

$$
\mathbf{X}_{\boldsymbol{\alpha}}=<\mathbf{g}, H_{\boldsymbol{\alpha}}>_{L^{2}\left(\boldsymbol{\Xi}, d P_{\boldsymbol{\xi}}\right)}=E\left(\mathbf{g}(\boldsymbol{\xi}) H_{\boldsymbol{\alpha}}(\boldsymbol{\xi})\right)=\int_{\boldsymbol{\Xi}} \mathbf{g}(\mathbf{y}) H_{\boldsymbol{\alpha}}(\mathbf{y}) d P_{\boldsymbol{\xi}}(\mathbf{y})
$$

where $E$ denotes the mathematical expectation. For the sake of simplicity, we here assume that the $\xi_{i}$ are independent identically distributed random variables, with probability law $P_{\xi}$ and support $\Xi \subset \mathbb{R}$. We then introduce a basis of orthonormal multidimensional polynomials. Letting $\mathcal{J}_{m}=\mathbb{N}^{m}$ be the set of m-dimensional multi-indices, the $H_{\boldsymbol{\alpha}}$ are defined by:

$$
H_{\boldsymbol{\alpha}}(\boldsymbol{\xi})=h_{\alpha_{1}}\left(\xi_{1}\right) \ldots h_{\alpha_{m}}\left(\xi_{m}\right)
$$

where the $\left\{h_{j}\right\}_{j \in \mathbb{N}}$ form an orthonormal polynomial basis of $L^{2}\left(\Xi, d P_{\xi}\right)$. For example, in the case where the $\xi_{i}$ are gaussian (resp. uniform) random variables, the $h_{j}$ are Hermite (resp. Legendre) polynomials [25]. The random vector is then approximated by truncating decomposition (9) on the generalized PC of degree $p$ :

$$
\mathbf{X}(\theta) \approx \sum_{\boldsymbol{\alpha} \in \mathcal{J}_{m, p}} \mathbf{X}_{\boldsymbol{\alpha}} H_{\boldsymbol{\alpha}}(\boldsymbol{\xi}(\theta))
$$

where $\mathcal{J}_{m, p}=\left\{\boldsymbol{\alpha} \in \mathcal{J}_{m}=\mathbb{N}^{m} ;|\boldsymbol{\alpha}| \leqslant p\right\}$.

\subsection{Maximum likelihood estimation}

4.2.1. Conservation of second order moments of samples As in [13], we impose the second order moments of samples to be conserved after the probabilistic identification. Although a few samples are available in practice, one can be relatively confident in those moments. In the following, we will denote the set of multi-indices $\mathcal{J}_{m, p}=\left\{\boldsymbol{\alpha}_{0}, \ldots, \boldsymbol{\alpha}_{P}\right\}$, with $P+1=\frac{(m+p) !}{m ! p !}$. As a convention, the first multi-index is defined by $\boldsymbol{\alpha}_{0}=(0, \ldots, 0)$ and is then associated with the constant polynomial $H_{\boldsymbol{\alpha}_{0}}=1$. Then, the conservation of the mean imposes $E(\mathbf{X})=\mathbf{X}_{\boldsymbol{\alpha}_{0}}=0$. Denoting $\mathbf{A}=\left(\mathbf{X}_{\boldsymbol{\alpha}_{1}} \ldots \mathbf{X}_{\boldsymbol{\alpha}_{P}}\right)^{T} \in \mathbb{R}^{P \times m}$ the matrix whose rows are the remaining chaos coefficients of $\mathbf{X}$, the conservation of covariance matrix imposes the following constraint on $\mathbf{A}$ :

$$
E\left(\mathbf{X X}^{T}\right)=\mathbf{A}^{T} \mathbf{A}=\mathbf{I}_{m}
$$

The identification of the coefficients is then equivalent to the identification of an orthogonal matrix $\mathbf{A} \in \mathbb{R}^{P \times m}$. The set of orthogonal matrices in $\mathbb{R}^{P \times m}$ is the compact Stiefel manifold, denoted $\mathbb{S}(P, m)$.

4.2.2. Maximum likelihood principle The likelihood function, for a given matrix of coefficients A, is defined by

$$
L(\mathbf{A})=\prod_{k=1}^{Q} p_{\mathbf{X}}\left(\mathbf{X}^{(k)} ; \mathbf{A}\right),
$$

where the $\mathbf{X}^{(k)}$ are the samples and $p_{\mathbf{X}}(\cdot ; \mathbf{A})$ the joint probability density function (pdf) of the chaos decomposition of $\mathbf{X}$. In practice, due to the prohibitive cost of the estimation of joint 
pdfs and also due to the small number of available samples, it was proposed in [13] to use a pseudo likelihood function:

$$
L(\mathbf{A})=\prod_{k=1}^{Q} \prod_{i=1}^{m} p_{X_{i}}\left(X_{i}^{(k)} ; \mathbf{A}\right)
$$

whose estimation only requires the evaluation of marginal pdfs. In practice, for numerical reasons, we rather use the opposite of the pseudo log-likelihood function:

$$
f(\mathbf{A})=-\log (L(\mathbf{A}))=-\sum_{k=1}^{Q} \sum_{i=1}^{m} \log \left(p_{X_{i}}\left(X_{i}^{(k)} ; \mathbf{A}\right)\right) .
$$

Finally, matrix $\mathbf{A}$ is defined by the following optimization problem on a compact Stiefel manifold:

$$
\mathbf{A}=\underset{\mathbf{A} \in \mathbb{S}(P, m)}{\operatorname{argmin}} f(\mathbf{A})
$$

Remark 2. When using a classical maximum likelihood estimation for parameters $\mathbf{A}$, a better result would be obtained by relaxing the constraint $\mathbf{A} \in \mathbb{S}(P, m)$, and letting $\mathbf{A} \in \mathbb{R}^{P \times m}$. However, when using the pseudo likelihood function, if this constraint is not imposed, the maximum likelihood principle is equivalent to uncoupled maximum principles for each random variable. Imposing the constraint then allows preserving an information on the dependency between variables.

Remark 3. Marginal pdfs are numerically computed with a kernel density estimation [26]: letting $\left\{\tilde{X}_{i}^{s}\right\}_{s=1}^{\tilde{Q}}$ be $\tilde{Q}$ independent samples of $X_{i}$, obtained from a chaos decomposition with given coefficients $\mathbf{A}$, we evaluate

$$
p_{X_{i}}\left(X_{i}^{(k)} ; \mathbf{A}\right) \approx \frac{1}{\tilde{Q}} \sum_{s=1}^{\tilde{Q}} \frac{1}{\delta} K\left(\frac{X_{i}^{(k)}-\tilde{X}_{i}^{s}}{\delta}\right)
$$

where $K$ is the Kernel function and $\delta$ the bandwidth.

4.2.3. Resolution of the optimization problem The resolution of optimization problem (15) is a relatively hard task due to the nature of the function $f$ and the possibly high dimensionality. In particular, $f$ may present local minima and it can be observed in practice that some local minima yield a bad probabilistic representation of $\mathbf{X}$. A global optimization procedure must then be provided. Dedicated algorithms, which could be used for our purpose, have been proposed in the literature in order to solve optimization problems on compact Stiefel manifolds [27]. Some of these algorithms preserve the specific geometry of the manifold, e.g. by taking into account the associated Riemannian metric in gradient algorithms.

Here, we propose to use a global random search algorithm in order to provide several initializations for a classical descent algorithm. Samples of the random search algorithm are generated with respect to the uniform probability measure on the Stiefel manifold (see e.g. [28]). This can be achieved by generating a sample $\widetilde{\mathbf{A}} \in \mathbb{R}^{P \times m}$ of a random matrix with independent identically distributed entries in $N(0,1)$ and then by computing $\mathbf{A}=\widetilde{\mathbf{A}}\left(\widetilde{\mathbf{A}}^{T} \widetilde{\mathbf{A}}\right)^{-1 / 2}$. In order to use a wider class of descent algorithms but also to reduce the dimension of the optimization problem, the problem can be reformulated as an unconstrained optimization problem. This 
is made possible by introducing a minimal parametrization of the compact Stiefel manifold $\mathbb{S}(P, m)$. Let us denote by $\varphi \in \mathbb{R}^{d}$ the set of parameters, where $d=P m-m(m+1) / 2$ is the dimension of the manifold, and by $T$ the mapping

$$
T: \boldsymbol{\varphi} \in \mathbb{R}^{d} \mapsto \mathbf{A}=T(\boldsymbol{\varphi}) \in \mathbb{S}(P, m),
$$

The unconstrained optimization problem can then be written:

$$
\varphi=\underset{\varphi \in \mathbb{R}^{d}}{\operatorname{argmin}} f(T(\varphi))
$$

A possible parametrization using matrix exponential is given in appendix II. In practice, we observe relatively good convergence properties of unconstrained optimization algorithms for problems where constrained optimization algorithms do not converge.

Remark 4. Function $f$ may be very non-smooth in some regions of $\mathbb{S}(P, m)$ and cause a non-convergence of optimization algorithms. In fact, these regions correspond to high values of $f$, irregularities coming from a bad numerical estimation of low values of likelihood. This evaluation can be improved by using more samples $\widetilde{Q}$ in the kernel estimation of the likelihood function. However, in practice, since we are not interested in these regions, we will prefer performing more global random search iterations for providing to the descent algorithm "well located" initializations.

Remark 5. It can be easily shown that function $f$ admits some symmetries with respect to coefficients $\mathbf{A}$. This comes from classical symmetries of PC expansions, for which different sets of coefficients can lead to the same probability laws of the resulting random vector. It follows that the global optimization problem (15) may admit several solutions.

5. Polynomial chaos representation for mutually independent random variables

The general maximum likelihood approach, requiring the resolution of (15), may be infeasible in practice due to the possible large number of parameters to be identified. In this section, we will assume that random variables $X_{i}$ are mutually independent. This strong hypothesis, which will be validated in numerical examples, seems reasonable in practice due to the small number of available samples. Each random variable can then be separately decomposed on a one-dimensional polynomial chaos basis of degree $p$ :

$$
X_{i}(\theta)=\sum_{\alpha=0}^{\infty} X_{i, \alpha} h_{\alpha}\left(\xi_{i}(\theta)\right) \approx \sum_{\alpha=0}^{p} X_{i, \alpha} h_{\alpha}\left(\xi_{i}(\theta)\right)
$$

where the $\xi_{i}$ are independent identically distributed random variables with support $\Xi \subset \mathbb{R}$ and probability law $P_{\xi}$, and where the $\left\{h_{\alpha}\right\}_{\alpha \in \mathbb{N}}$ form an orthonormal polynomial basis of $L^{2}\left(\Xi, d P_{\xi}\right)$. We then propose two possible strategies for the identification of one-dimensional chaos decompositions. The first one is the maximum likelihood estimation described in the previous section, which is here greatly simplified when applied to independent realvalued random variables. The second one is a projection method using empirical cumulative distribution functions of samples. The latter method leads to a very fast computation of the decomposition. 


\subsection{Maximum likelihood estimation}

The conservation of mean and variance of samples impose to the set of chaos coefficients the following constraints: $X_{i, 0}=0$ and $\sum_{\alpha=1}^{p} X_{i, \alpha}^{2}=1$. The undetermined chaos coefficients $\mathbf{a}_{i}=\left(X_{i, 1}, \ldots, X_{i, p}\right)^{T}$ can then be defined by the maximum likelihood principle:

$$
\mathbf{a}_{i}=\underset{\mathbf{a} \in \mathbb{S}(p, 1)}{\operatorname{argmin}} f_{i}(\mathbf{a})
$$

where $\mathbb{S}(p, 1)$ is the hypersphere in $\mathbb{R}^{p}$ and

$$
f_{i}(\mathbf{a})=-\sum_{k=1}^{Q} \log \left(p_{X_{i}}\left(X_{i}^{(k)} ; \mathbf{a}\right)\right)
$$

For solving problem (18), we use the same numerical procedure as described in section 4.2.3, with a minimal parametrization of the hypersphere detailed in appendix II.

\subsection{A projection method using empirical cumulative distribution functions}

The second order random variable $X_{i}$ can be expressed in terms of random variable $\xi_{i}$ by the following non-linear mapping:

$$
X_{i}=F_{X_{i}}^{-1} \circ F_{\xi}\left(\xi_{i}\right)
$$

where $F_{X_{i}}$ (resp. $F_{\xi}$ ) denotes the cumulative distribution function (CDF) of $X_{i}$ (resp. $\xi_{i}$ ). The coefficients of the chaos expansion (17) are then defined as the projection of function $F_{X_{i}}^{-1} \circ F_{\xi}$ on orthonormal chaos basis functions:

$$
X_{i, \alpha}=<X_{i}, h_{\alpha}>_{L^{2}\left(\Xi, d P_{\xi}\right)}=E\left(X_{i} h_{\alpha}\left(\xi_{i}\right)\right)=\int_{\Xi} F_{X_{i}}^{-1}\left(F_{\xi}(y)\right) h_{\alpha}(y) d P_{\xi}(y) .
$$

Of course, function $F_{X_{i}}$ is not known. However, this function can be estimated by the empirical CDF. We denote by $\widetilde{F}_{X_{i}}$ the empirical CDF of $X$, estimated from samples: $\widetilde{F}_{X_{i}}(x)=\frac{1}{Q} \sum_{k=1}^{Q} I\left(X_{i}^{(k)} \leqslant x\right)$, where $I(A)$ is the indicator function of event $A$. We then introduce the following approximation $F_{X_{i}}^{-1} \approx \widetilde{F}_{X_{i}}^{-1}$, where $\widetilde{F}_{X_{i}}^{-1}:[0,1] \rightarrow \mathbb{R}$ is uniquely defined as $\widetilde{F}_{X_{i}}^{-1}(y)=\min \left\{x \in\left\{X^{(k)}\right\}_{k=1}^{Q} ; \widetilde{F}_{X_{i}}(x) \geqslant y\right\}$. Then, the coefficients of the chaos expansion can be approximated using a numerical integration:

$$
X_{i, \alpha} \approx \int_{\Xi} \widetilde{F}_{X_{i}}^{-1}\left(F_{\xi}(y)\right) h_{\alpha}(y) d P_{\xi}(y) \approx \sum_{k=1}^{N_{g}} \omega_{k} \widetilde{F}_{X_{i}}^{-1}\left(F_{\xi}\left(y_{k}\right)\right) h_{\alpha}\left(y_{k}\right)
$$

where the $\left\{\omega_{k}, y_{k}\right\}_{k=1}^{N_{g}}$ are integration weights and points. In practice, we will use a Gauss quadrature associated with measure $P_{\xi}$. For example, if $\xi_{i}$ is a Gaussian (resp. uniform) random variable, it leads to the classical Gauss-Hermite (resp. Gauss-Legendre) quadrature, where the integration points are the roots of the Hermite (resp. Legendre) polynomial of degree $N_{g}$.

As we will see in numerical examples, the decompositions obtained by this projection technique and by the maximum likelihood method are very similar. However, the proposed projection technique leads to a very fast identification of the chaos decomposition, when compared to maximum likelihood estimation. 


\section{Numerical examples}

\subsection{Description of the overall procedure}

Before proceeding to the numerical examples presented in this section, a summary of the overall identification procedure is given for clarity reasons. The starting point is a collection of contrasted images $\left\{I^{(k)}\right\}_{k=1}^{Q}$, representing $Q$ samples of a random shape. Then, the overall procedure consists in the following steps:

- Step 1: Shape recovery with the level-set technique: construction of a collection of discretized level-set functions $\left\{\phi^{(k)}\right\}_{k=1}^{Q}$, associated with the collection of images $\left\{I^{(k)}\right\}_{k=1}^{Q}$,

- Step 2: Reduction of information through empirical Karhunen-Loève expansion: a levelset sample is decomposed as follows

$$
\phi^{(k)} \approx \mu_{\boldsymbol{\phi}}+\sum_{i=1}^{m} \widetilde{\mathbf{U}}_{i} X_{i}^{(k)}
$$

where the $\left\{\widetilde{\mathbf{U}}_{i}\right\}_{i=1}^{m}$ form an optimal reduced basis of $m$ deterministic vectors and the $\left\{X_{i}^{(k)}\right\}_{i=1}^{m}$ are the associated samples of random components,

- Step 3: Probabilistic identification of random vector $\mathbf{X}=\left(X_{1}, \ldots, X_{n}\right)$ from samples $\mathbf{X}^{(k)}=\left(X_{1}^{(k)}, \ldots, X_{n}^{(k)}\right)$ : decomposition on a polynomial chaos basis of degree $p$ in dimension $m$

$$
\mathbf{X}=\sum_{\alpha \in \mathcal{J}_{m, p}} \mathbf{X}_{\alpha} H_{\alpha}(\boldsymbol{\xi})
$$

with two alternatives for the identification of coefficients:

- Without independence hypothesis: maximum likelihood estimation ML(D) (optimization problem on a Stiefel manifold)

- With independence hypothesis: maximum likelihood estimation ML(I) (optimization on a hypersphere) or projection method based on the empirical cumulative distribution function of samples $\operatorname{EP}(\mathrm{I})$.

In the following subsections, three numerical examples are provided in order to illustrate the accuracy and efficiency of the proposed methodology. It has to be noted that the numerical investigation presented here is based on "manufactured" problems: in the first two examples, the different outcomes of the random shape to identify are samples of an analytically defined random level-set (only steps 2 and 3 are performed) while in the third example, sample images are artificially generated from an analytically defined random level-set. The overall procedure (steps 1 to 3 ) is then validated on this third example.

\subsection{Example 1: random rough circle}

In the first example, the random shape to identify is a "rough" circle (see Figure 3) whose boundary is a random curve defined by:

$$
C(\theta)=\left\{\boldsymbol{c}+R(\alpha, \theta)\left(\cos (\alpha) \boldsymbol{e}_{1}+\sin (\alpha) \boldsymbol{e}_{2}\right), \alpha \in[0,2 \pi]\right\},
$$


where $\boldsymbol{c}$ is the "center", $\boldsymbol{e}_{1}$ and $\boldsymbol{e}_{2}$ are the unit vectors of the Cartesian coordinate system and $R$ is a random field indexed by the polar angle $\alpha$ of the polar coordinate system centered at c.

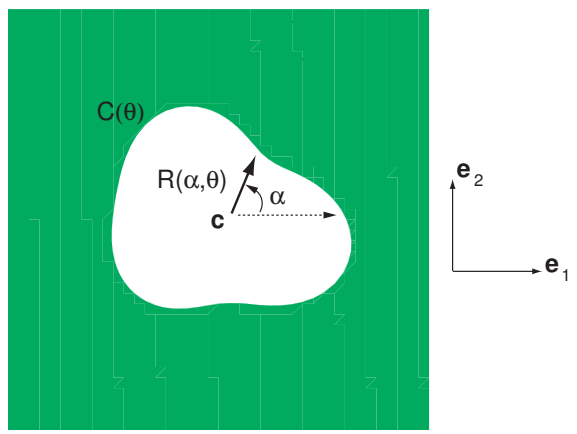

Figure 3. Schematic representation of the rough circle of Eq. (22).

Random field $R$ is defined by:

$$
\begin{aligned}
& R(\alpha, \theta)=0.2+0.03 Y_{1}(\theta)+ \\
& \quad 0.015\left(Y_{2}(\theta) \cos \left(k_{1} \alpha\right)+Y_{3}(\theta) \sin \left(k_{1} \alpha\right)+Y_{4}(\theta) \cos \left(k_{2} \alpha\right)+Y_{5}(\theta) \sin \left(k_{2} \alpha\right)\right)
\end{aligned}
$$

where $k_{1}$ and $k_{2}$ are deterministic constants and $\left(Y_{1}(\theta), \ldots, Y_{5}(\theta)\right)$ are independent identically distributed (i.i.d.) uniform random variables: $Y_{i} \in U(-\sqrt{3}, \sqrt{3}), i=1 \ldots 5$. The corresponding level-set is taken as the signed radial distance function to the curve $C(\theta)$. It can be explicitly written as $\phi(\boldsymbol{x}, \theta)=\|\boldsymbol{x}-\boldsymbol{c}\|-R(\alpha(\boldsymbol{x}), \theta)$ and is defined in a square domain $[0,1] \times[0,1]$. The random rough circle of Eq. (22) is clearly the iso-zero of level-set $\phi$.

$Q=100$ samples of the random level-set will be the starting point of the numerical investigation. Use of a limited number of samples is made because, in practice, experimental campaigns or in-site measurements are often very expensive and thus only a few samples of the random geometry to identify are available. In this first example, a Legendre PC will be used for the representation of the random variables resulting from K-L decomposition.

Due to the specific form of the random level-set function, its exact K-L decomposition in 5 modes can be obtained in this case. In addition, the fact that the random variables to identify are statistically independent permits a straightforward comparison of the accuracy of the alternative techniques used for the identification of the PC coefficients (see sections 4 and $5)$. The pseudo log-likelihood function, the pdf of the initial (resulting from K-L expansion) and identified random variables as well as the error in the probability $P_{i n}(\boldsymbol{x})$ to be inside the hole are used as error criteria in the comparison. The probability $P_{i n}(\boldsymbol{x})$ is defined by

$$
P_{\text {in }}(\boldsymbol{x})=P(\phi(\boldsymbol{x}, \theta)<0),
$$

and can be evaluated from samples as follows:

$$
P_{\text {in }}(\boldsymbol{x}) \approx \frac{1}{Q} \operatorname{Card}\left\{k \in\{1 \ldots Q\} ; \phi^{(k)}(\boldsymbol{x})<0\right\} .
$$

In Figure 4, the values of the pseudo log-likelihood calculated for the initial and identified random variables are plotted as a function of the order $p$ of Legendre PC decomposition using the three identification alternatives. 


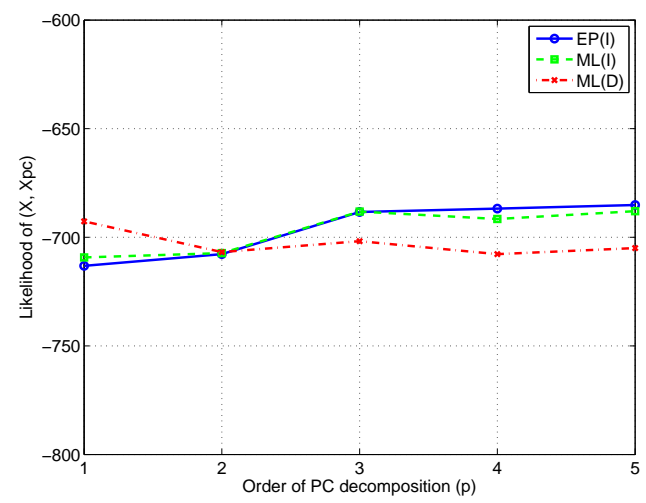

Figure 4. Example 1: Pseudo log-likelihood values of the initial and identified random variables as a function of the order $p$ of Legendre PC decomposition.

It appears that for orders $p \geqslant 3$, better results are obtained with the techniques assuming independence between the random variables. The results of empirical projection (denoted as $\mathrm{EP}(\mathrm{I})$ ) and maximum likelihood method with independence hypothesis (denoted as $\mathrm{ML}(\mathrm{I})$ ) practically coincide. The fact that these two methods assuming independence between variables give satisfactory results is rational due to the definition of the specific problem. However, better results would have been expected for the maximum likelihood approach without independence hypothesis (denoted as $\mathrm{ML}(\mathrm{D})$ ). In fact, the bad results for $p \geqslant 3$ are due to a bad resolution of the corresponding maximum likelihood optimization problem, which is a very hard highdimensional optimization problem. The superiority of $\operatorname{EP}(\mathrm{I})$ and $\mathrm{ML}(\mathrm{I})$ against $\mathrm{ML}(\mathrm{D})$ is confirmed by the two other error criteria used in this study.

Figure 5 depicts the marginal pdf of the initial and identified variables obtained using the three identification techniques for Legendre chaos with $p=1$ and the $\operatorname{EP}(\mathrm{I})$ technique with $p=3$. The pdfs resulting from the $\mathrm{EP}(\mathrm{I})$ and $\mathrm{ML}(\mathrm{I})$ techniques are clearly better. The results slightly improve for higher order $p$ in the PC series, as shown in Figures 5 and 6 (for $p=3$, the error in $P_{i n}$ is about 0.05$)$. At this point, it must be noted that the $\operatorname{EP}(\mathrm{I})$ method has the smallest computational cost compared to the two other techniques. It is about two orders of magnitude faster than the $\mathrm{ML}(\mathrm{I})$ method and about three orders of magnitude faster than the $\mathrm{ML}(\mathrm{D})$ approach.

To summarize, in the example of the random rough circle involving a few independent random variables to identify, the projection method gives the best results in both terms of accuracy and computational efficiency. In practical geometry identification problems, it is in general more likely to assume statistical dependence between the random variables characterizing the shape to identify. However, the assumption of independence is very often used in practice because it is consistent with the fact that a small collection of images is usually available. The effect of independence hypothesis on the identification of random shapes involving dependent random variables is illustrated with the next numerical example. 


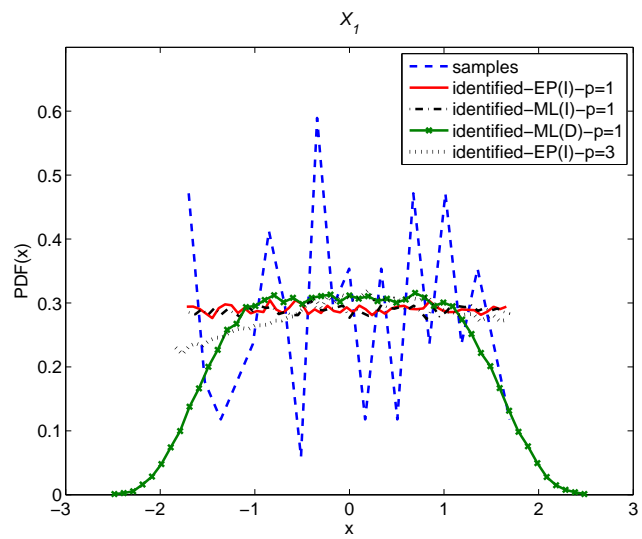

(a)

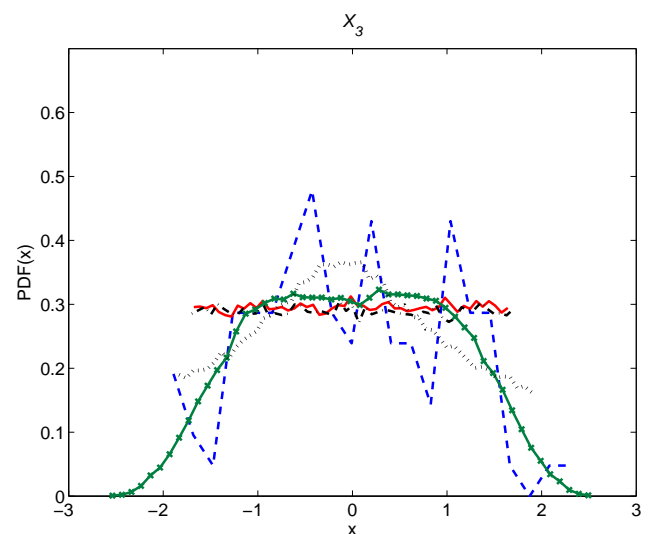

(c)

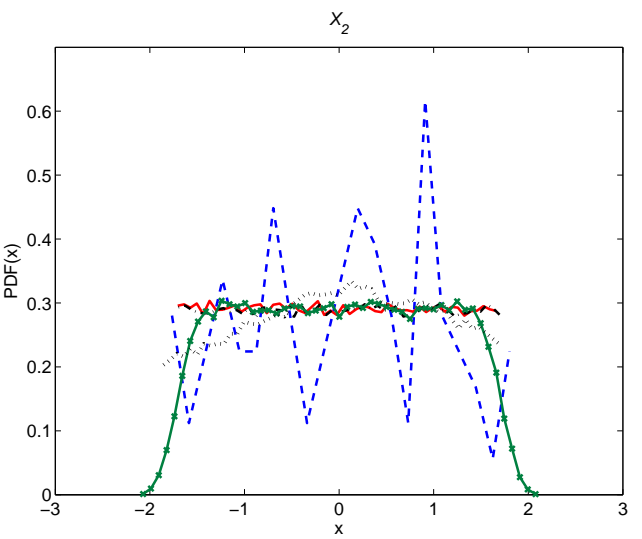

(b)

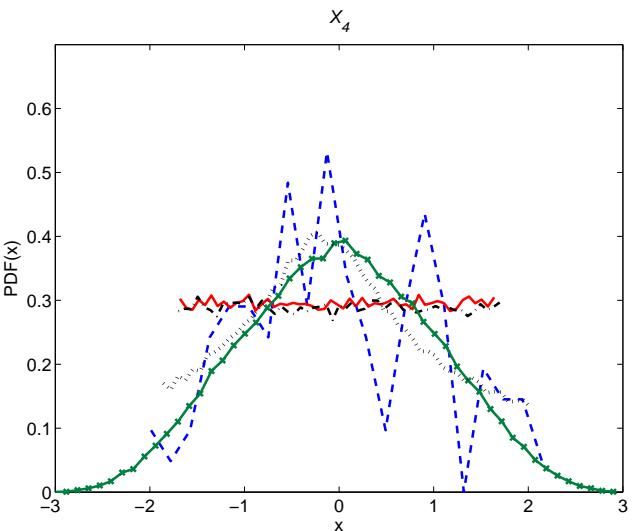

(d)

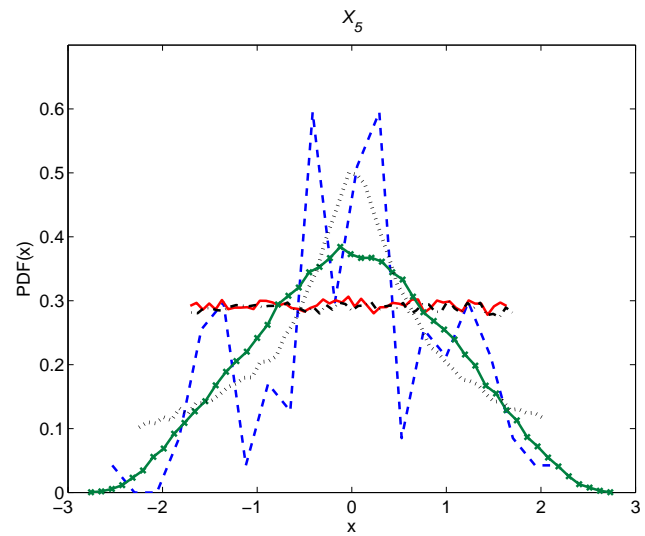

(e)

Figure 5. Example 1: Marginal pdf of the initial and identified random variables (Legendre chaos). 


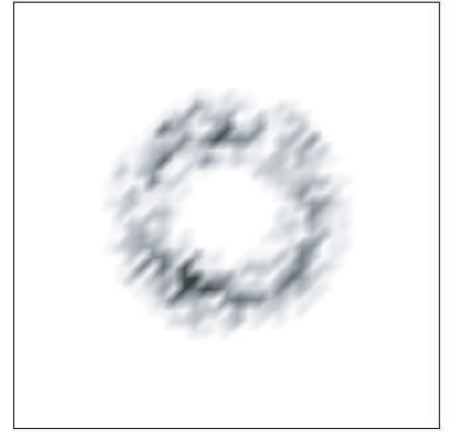

(a)

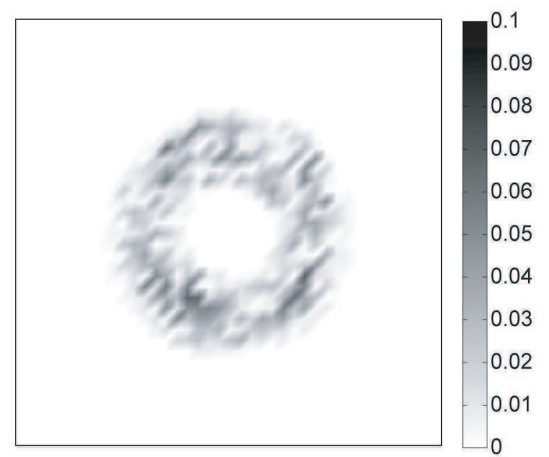

(b)

Figure 6. Example 1: Error in the probability to be inside the hole (EP(I), Legendre chaos) with (a) $p=1$ and (b) $p=3$.

\subsection{Example 2: circle with random position of center and random radius}

In the second example, a circle with random position of center and random radius is considered. The location of the center of the circle $\boldsymbol{c}=\left(c_{1}, c_{2}\right)$ is assumed to depend on two statistically independent uniform random variables $c_{1}(\theta) \in U(0.4,0.6)$ and $c_{2}(\theta) \in U(0.4,0.6)$. Its radius is also modeled with a uniform random variable $R(\theta) \in U(0.2,0.3)$, which is statistically independent of $c_{1}$ and $c_{2}$. The corresponding level-set function can be explicitly written as $\phi(\boldsymbol{x}, \theta)=\|\boldsymbol{x}-\boldsymbol{c}(\theta)\|-R(\theta)$ and is again defined in a square domain $[0,1] \times[0,1] . Q=100$ samples of the random level-set will again be the starting point of the numerical investigation. Two types of PC decomposition, Legendre and Hermite, will be used for the representation of the random variables resulting from K-L decomposition. The same error criteria as in Example 1 will be used to quantify the accuracy of the different approaches.

Using a tolerance $\epsilon_{K L}$ equal to 0.01 in Eq. (7) leads to a K-L decomposition of the random level-set in 12 terms and thus 12 (uncorrelated but dependent) random variables have to be identified in this case. The effect of the value of tolerance $\epsilon_{K L}$ on the reconstruction of the random level-set is quantified using iso-contour plots of the probability $P_{i n}$ to be inside the hole, defined in equation (24). In Figure 7, three contours are plotted $\left(P_{\text {in }}=0.1,0.5\right.$ and 0.9$)$ for three different values of tolerance $\epsilon_{K L}$. The effect of $\epsilon_{K L}$ on the iso-probability plots is obvious. It can also be stated that the value of tolerance $\left(\epsilon_{K L}=0.01\right)$ used in this example leads to a satisfactory reconstruction of the probabilistic content of the level-sets.

In Figure 8, the values of the pseudo log-likelihood calculated for the initial and identified random variables are plotted as a function of the order $p$ of $\mathrm{PC}$ decomposition using the three identification alternatives and the two types of PC (Legendre and Hermite). It must be noted that, in the case of the ML(D) approach, values of the pseudo log-likelihood up to $p=3$ have been calculated due to the prohibitive computational cost required for higher order PC expansions. A faster convergence with $p$ is observed for the Hermite chaos. For low order Legendre chaos $(p \leqslant 3)$, better results in likelihood are obtained with the ML(D) technique indicating that the dependence hypothesis is more suitable in this case. However, the results 


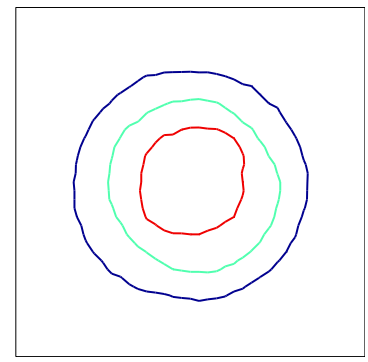

(a)

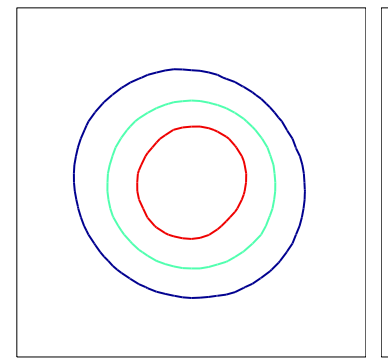

(c)

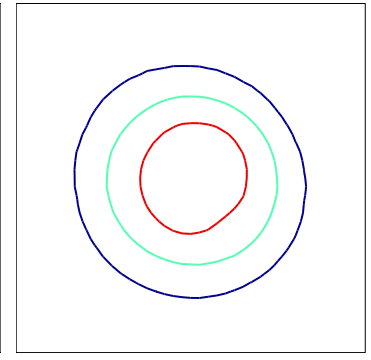

(b)

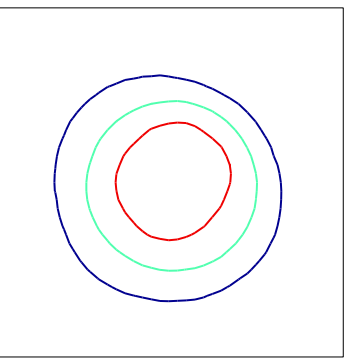

(d)

Figure 7. Example 2: Iso-contour plots of $P_{\text {in }}$ (blue $=0.1$, light blue $=0.5$ and red $=0.9$ ) for Legendre chaos and $p=3$ : (a) initial level-sets, (b) $\epsilon_{K L}=0.01$, (c) $\epsilon_{K L}=0.1$, (d) $\epsilon_{K L}=0.5$.

obtained with the techniques assuming independence improve for larger values of $p$ and thus these techniques can provide a very efficient alternative to ML(D). Similar observations can be made in the case of Hermite chaos with the difference that ML(D) leads now to slightly worse results. This can be attributed to a bad solution of the corresponding optimization problem.

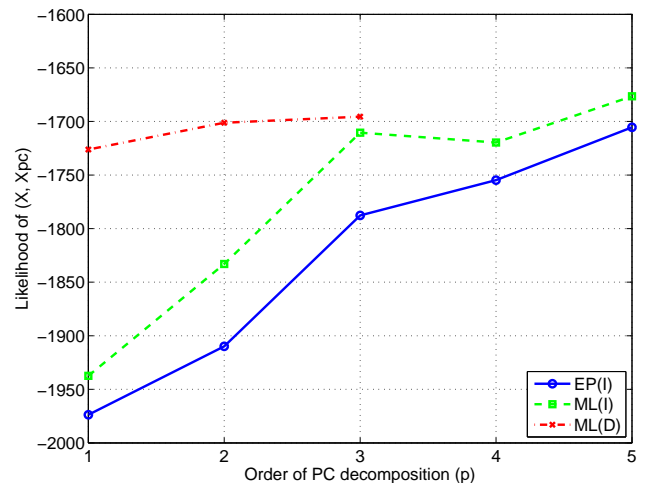

(a)

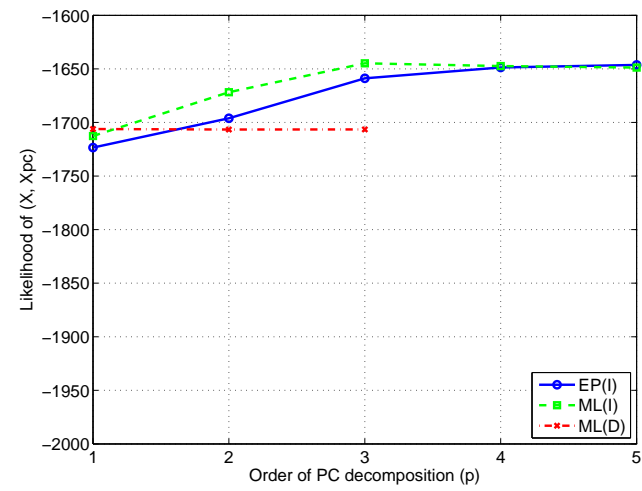

(b)

Figure 8. Example 2: Pseudo log-likelihood values of the initial and identified random variables as a function of the order $p$ of PC decomposition: (a) Legendre chaos, (b) Hermite chaos. 
Figure 9 shows the marginal pdf of the first 4 initial and identified variables obtained using $\mathrm{EP}(\mathrm{I})$ and $\mathrm{ML}(\mathrm{D})$ for Legendre and Hermite chaos of order $p=3$. Although the exact pdf of the random variables is not known in this example, it seems that a better matching of the sample pdf is achieved with the EP(I) technique for Legendre chaos. Concerning the error in the probability to be inside the hole $P_{i n}$, it again decreases as the PC order grows up. The errors of the three identification alternatives are comparable (Figure 10). However, the smallest error is not provided by the ML(D) approach.

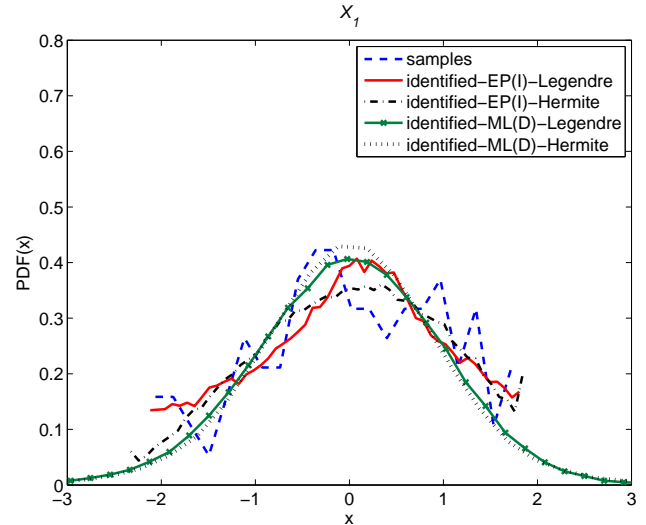

(a)

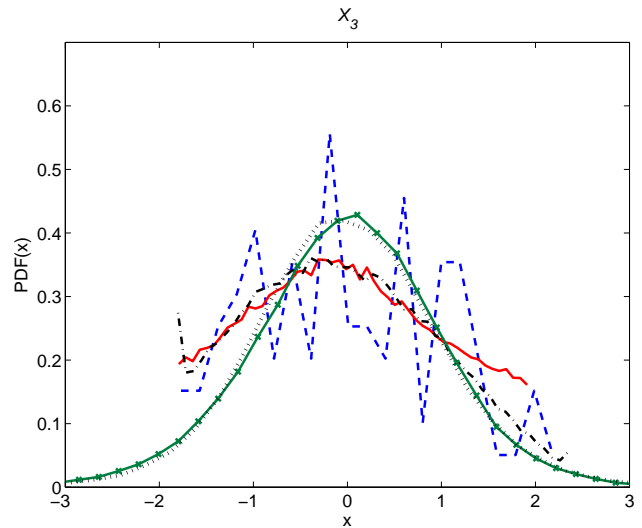

(c)

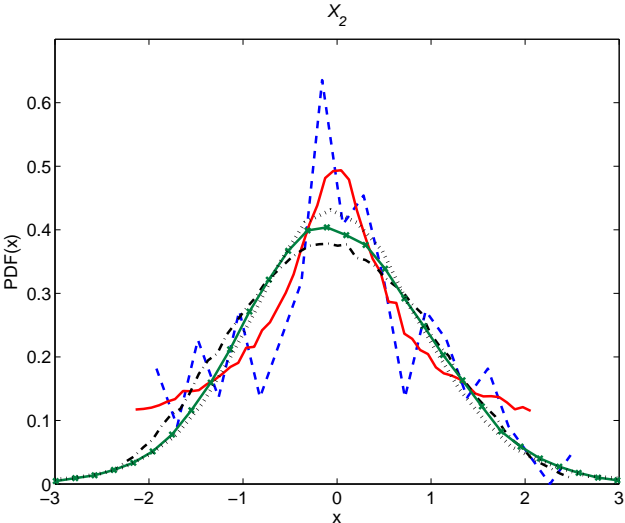

(b)

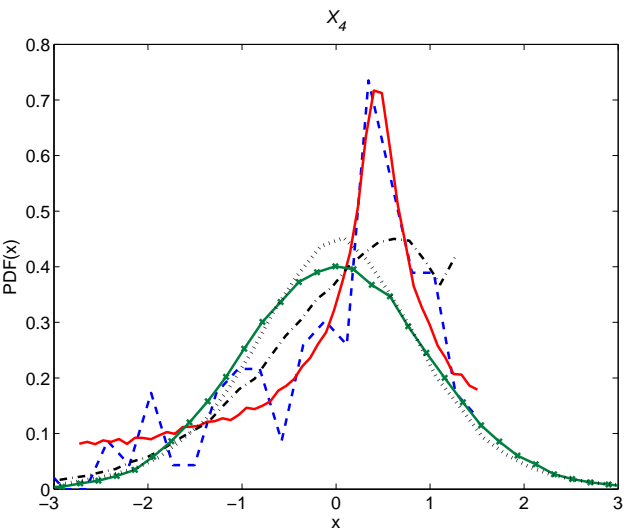

(d)

Figure 9. Example 2: Marginal pdf of the first 4 initial and identified random variables $(p=3)$.

To summarize, for the circle with random position of center and random radius examined in this example, the three identification alternatives seemed to provide comparably accurate results in the identification of the random variables resulting from the $\mathrm{K}-\mathrm{L}$ decomposition. However, the two techniques assuming independence are very competitive in terms of accuracy and much better in terms of computational efficiency than the ML(D) approach. 


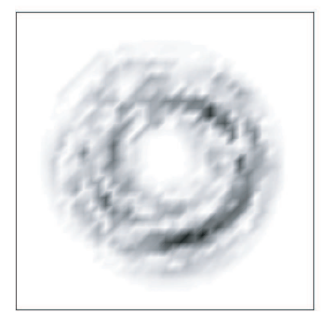

(a)

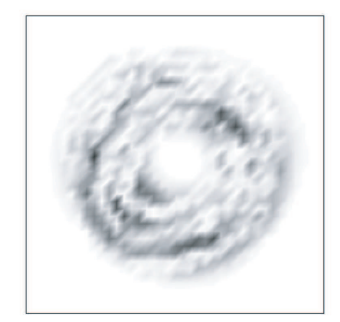

(b)

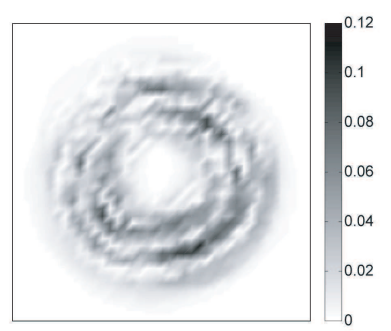

(c)

Figure 10. Example 2: Error in the probability to be inside the hole (Legendre chaos, $p=3$ ): (a) $\operatorname{EP}(\mathrm{I}),(\mathrm{b}) \mathrm{ML}(\mathrm{I})$ and (c) ML(D).

\subsection{Example 3: rough circle recovered from sample images}

In this example, the samples of the random shape to identify are obtained using the shape recovery technique based on the level-set method described in section 2 of the paper. The starting point for the identification procedure is a set of $Q=100$ images, which are artificially generated from the analytically defined level-set function given in Example 1. With an appropriate choice of the speed of the front (see Eq. (2)) and by applying 100 times the shape recovery technique to the initial set of sample images, the corresponding set of 100 discretized level-sets to identify is obtained. It is worth noting that, since the recovery procedure involves calculation of image gradients whose accuracy depends on the mesh size, a sufficiently fine mesh must be used for the recovery in order to be able to capture details of shape features. In our case, a $100 \times 100$ mesh has been used to this purpose. Figure 11 illustrates the filtered gradients of three sample images $I^{(k)}$ and the corresponding iso-zero of the recovered level-sets.

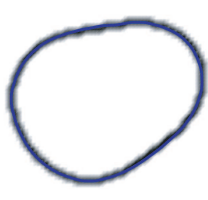

(a)

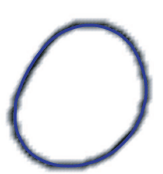

(b)

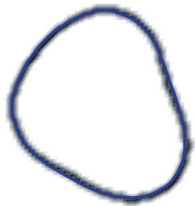

(c)

Figure 11. Example 3: Gradient intensity of three filtered sample images and corresponding iso-zero of recovered level-sets.

Legendre and Hermite chaos are used for the representation of the random variables resulting from K-L decomposition of the recovered level-sets. The accuracy of the different approaches is assessed through the error criteria introduced in the previous examples. Using a tolerance $\epsilon_{K L}$ equal to 0.05 in Eq. (7) leads to a K-L decomposition of the sample level-sets in 8 terms and thus 8 random variables have to be identified in this case. The effect of the recovery procedure and of the K-L decomposition on the reconstruction of the images is illustrated in Figure 12 
using iso-contour plots of $P_{i n}(\boldsymbol{x})$ (iso-contours $P_{i n}(\boldsymbol{x})=0.1,0.5$ and 0.9 ). It can be observed that the recovery procedure is sufficiently accurate and that the value of tolerance used in this example leads to a satisfactory reconstruction of the probabilistic content of the recovered images.

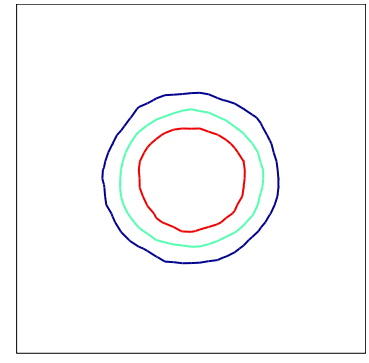

(a)

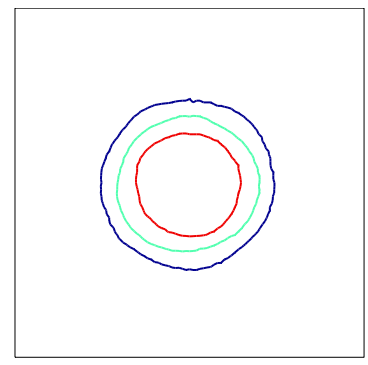

(b)

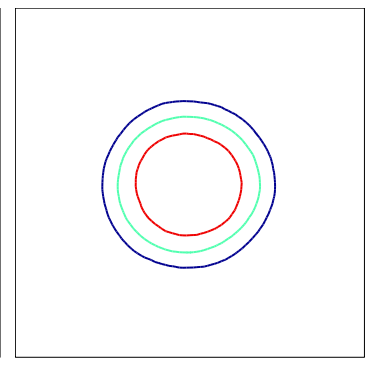

(c)

Figure 12. Example 3: Iso-contour plots of $P_{i n}$ (blue $=0.1$, light blue $=0.5$ and red $\left.=0.9\right)$ for Legendre chaos and $p=3$ : (a) initial images, (b) recovered level-sets and (c) recovered level-sets with K-L decomposition, $\epsilon_{K L}=0.05$.

In Figure 13, the values of the pseudo log-likelihood calculated for the initial and identified random variables are plotted as a function of the order $p$ of $\mathrm{PC}$ decomposition using the three identification alternatives and the two types of PC.

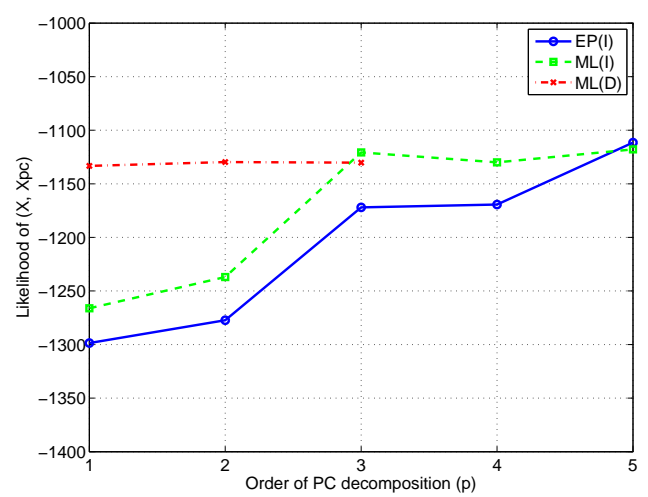

(a)

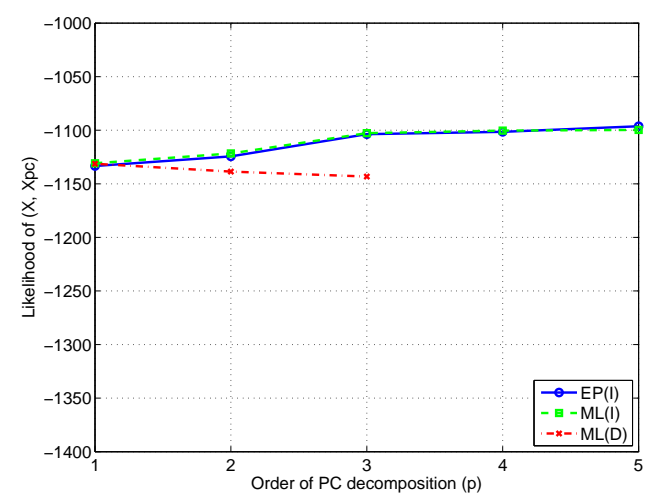

(b)

Figure 13. Example 3: Pseudo log-likelihood values of the initial and identified random variables as a function of the order $p$ of PC decomposition: (a) Legendre chaos, (b) Hermite chaos.

As in Example 2, values of the pseudo log-likelihood up to $p=3$ have been calculated in the case of the $\mathrm{ML}(\mathrm{D})$ approach due to the very large computational cost required for higher order PC expansion. For Hermite PC, the results obtained with $\mathrm{EP}(\mathrm{I})$ and $\mathrm{ML}(\mathrm{I})$ practically coincide and are clearly better than those of $\mathrm{ML}(\mathrm{D})$. For low order Legendre PC, the ML(D) technique gives better results in likelihood but the results of the two other methods substantially improve as $p$ grows up. This fact implies that the independence assumption is 
suitable to this problem and has not been strongly affected by the recovery procedure. In the Legendre PC case, the convergence is slower than in Example 1 due to the greater complexity of the problem. The marginal pdfs of the first 4 random variables shown in Figure 14 are similar to those of Example 1 (close to the uniform) confirming that the recovery procedure was sufficiently accurate. Similar results to those of Example 1 have also been obtained for the error in the probability to be inside the hole, presented in Figure 15.

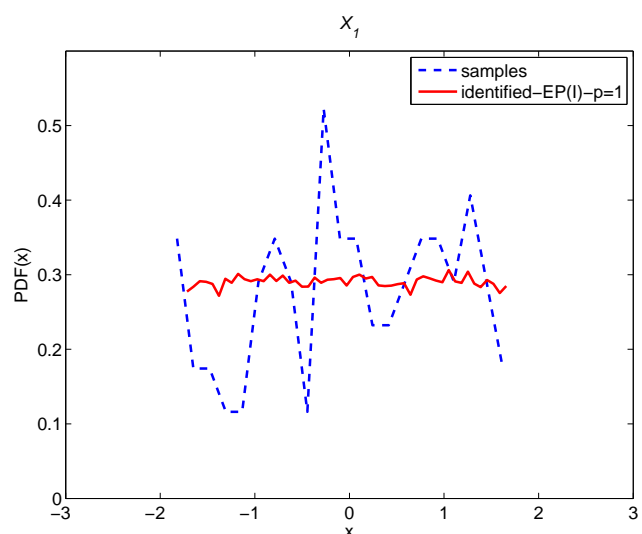

(a)

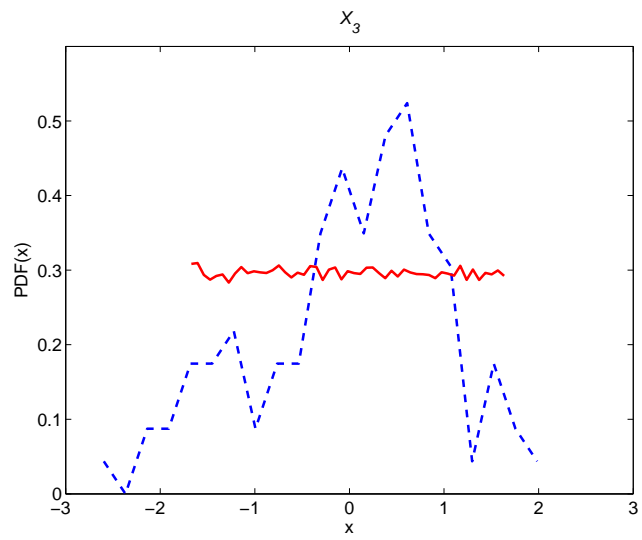

(c)

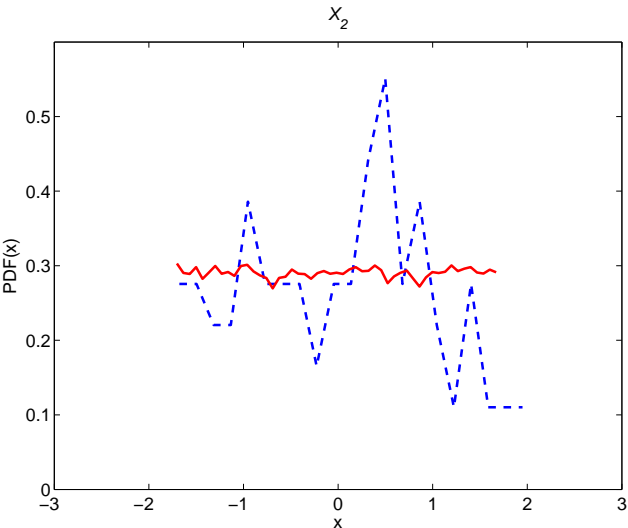

(b)

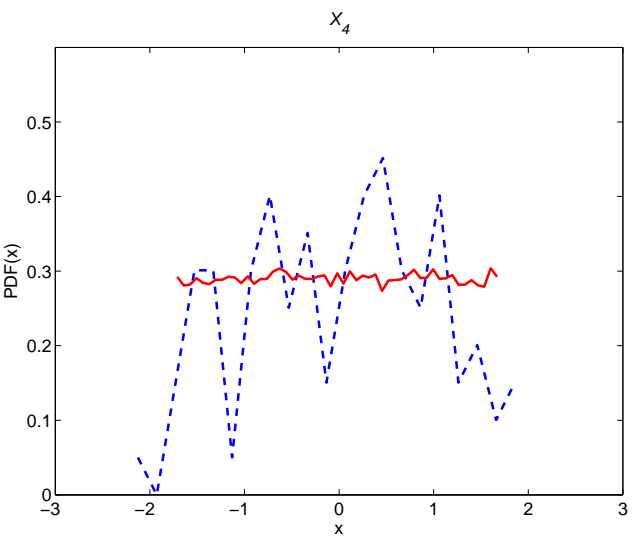

(d)

Figure 14. Example 3: Marginal pdf of the first 4 initial and identified random variables (Legendre chaos, $p=1, \operatorname{EP}(\mathrm{I}))$.

Before closing this section, it can again be stated that the two techniques assuming independence are very competitive in terms of accuracy compared to the ML(D) approach, while the projection method remains the approach having the smallest computational cost for the identification procedure. 


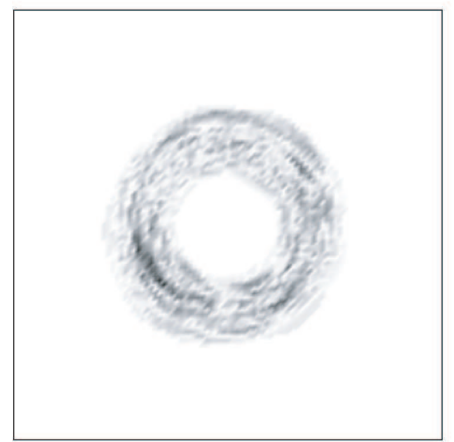

(a)

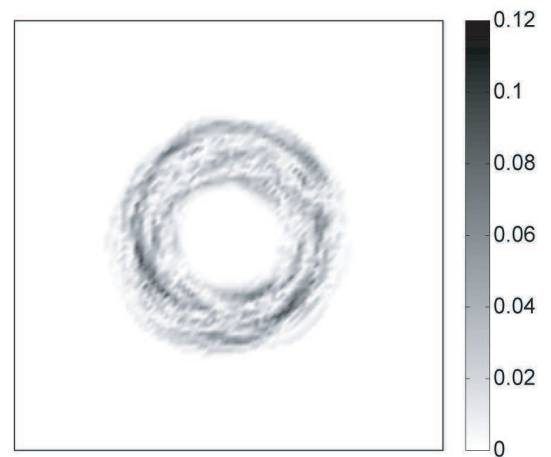

(b)

Figure 15. Example 3: Error in the probability to be inside the hole (EP(I), Legendre chaos) with (a) $p=1$ and (b) $p=3$.

7. Propagation of uncertainties in structural analysis

\subsection{Problem statement}

As an illustration of the propagation of geometrical uncertainties in structural analysis, we consider a linear elasticity problem defined on a random domain $\Omega(\boldsymbol{\xi})$, where $\boldsymbol{\xi}$ denotes the basic random variables describing the uncertainties on the geometry of the domain. These random variables define a finite dimensional probability space denoted $\left(\boldsymbol{\Xi}, \mathcal{B}_{\boldsymbol{\Xi}}, P_{\boldsymbol{\xi}}\right)$. We denote by $\boldsymbol{u}$ the displacement field, $\boldsymbol{\varepsilon}(\boldsymbol{u})$ the symmetric part of the displacement gradient (i.e. the strain tensor), and $\mathbf{C}$ the Hooke elasticity tensor. Homogeneous Dirichlet boundary conditions are imposed on a deterministic part $\Gamma_{1}$ of the boundary. Surface loads $\boldsymbol{F}$ are applied on another part $\Gamma_{2}$ of the boundary. For the sake of simplicity, we consider that $\Gamma_{2}$ is also deterministic. The problem is then to find $\boldsymbol{u}$ such that we have almost surely:

$$
\begin{aligned}
& \operatorname{div}(\mathbf{C}: \boldsymbol{\varepsilon}(\boldsymbol{u}))=0 \quad \text { on } \quad \Omega(\boldsymbol{\xi}) \\
& \boldsymbol{u}=0 \quad \text { on } \quad \Gamma_{1} \\
& (\mathbf{C}: \boldsymbol{\varepsilon}(\boldsymbol{u})) \cdot \boldsymbol{n}=\boldsymbol{F} \quad \text { on } \quad \Gamma_{2}
\end{aligned}
$$

\section{2. eXtended Stochastic Finite Element Method (X-SFEM)}

The problem is defined on a fictitious domain $D$ which contains all outcomes of the physical random domain $\Omega(\boldsymbol{\xi})$. The random domain is then implicitly defined by the level-set function $\phi(\boldsymbol{x}, \boldsymbol{\xi})$, which has been identified by the above procedure. By convention, we suppose that the level-set function takes negative values on the physical domain:

$$
\Omega(\boldsymbol{\xi})=\{\boldsymbol{x} \in D ; \phi(\boldsymbol{x}, \boldsymbol{\xi})<0\}
$$

Problem (25) is then reformulated by considering prolongation of functions on the fictitious domain. The random solution $\boldsymbol{u}$ is searched in function space $\mathcal{S} \otimes$, where $\mathcal{V}=\{\boldsymbol{v} \in$ $H_{1}(D)^{2} ; \boldsymbol{v}=0$ on $\left.\Gamma_{1}\right\}$ and $\mathcal{S}=L^{2}\left(\boldsymbol{\Xi}, d P_{\boldsymbol{\xi}}\right)$ is the space of second order random variables defined on $\boldsymbol{\Xi}$. A variational formulation of problem (25) can be written: find $\boldsymbol{u} \in \mathcal{V} \otimes \mathcal{S}$ such 
that

$$
A(\boldsymbol{u}, \boldsymbol{v})=B(\boldsymbol{v}), \quad \forall \boldsymbol{v} \in \mathcal{V} \otimes \mathcal{S}
$$

where $A$ and $B$ are bilinear and linear forms, defined by

$$
\begin{aligned}
& A(\boldsymbol{u}, \boldsymbol{v})=\int_{\Xi} \int_{D} \boldsymbol{\varepsilon}(\boldsymbol{u}): \boldsymbol{C}: \boldsymbol{\varepsilon}(\boldsymbol{v}) \mathcal{H}(-\phi) d \boldsymbol{x} d P_{\boldsymbol{\xi}}, \\
& B(\boldsymbol{v})=\int_{\Xi} \int_{\Gamma_{2}} \boldsymbol{v} \cdot \boldsymbol{F} d s d P_{\boldsymbol{\xi}},
\end{aligned}
$$

where $\mathcal{H}$ is the heaviside function $(\mathcal{H}(t)=1$ for $t>0, \mathcal{H}(t)=0$ for $t \leqslant 0)$. We then introduce a finite element function space $\mathcal{V}_{n} \subset \mathcal{V}$ associated with a fixed mesh of $D$ and a finite dimensional approximation space $\mathcal{S}_{p} \subset \mathcal{S}$ at the stochastic level (e.g. polynomial chaos of degree $p$ in dimension $m$. Note that the degree $p$ can be different from the one used for the decomposition of $\phi$ ). It then defines a tensor product approximation space:

$$
\mathcal{V}_{n} \otimes \mathcal{S}_{p}=\left\{\boldsymbol{v}(\boldsymbol{x}, \boldsymbol{\xi})=\sum_{i=1}^{n} \sum_{\alpha \in \mathcal{J}_{m, p}} v_{i, \alpha} \boldsymbol{\varphi}_{i}(\boldsymbol{x}) H_{\alpha}(\boldsymbol{\xi}), \boldsymbol{\varphi}_{i} \in \mathcal{V}, H_{\alpha} \in \mathcal{S}\right\}
$$

Galerkin approximate solution $\boldsymbol{u}_{n, p} \in \mathcal{V}_{n} \otimes \mathcal{S}_{p}$ is then defined by

$$
A\left(\boldsymbol{u}_{n, p}, \boldsymbol{v}_{n, p}\right)=B\left(\boldsymbol{v}_{n, p}\right), \quad \forall \boldsymbol{v}_{n, p} \in \mathcal{V}_{n} \otimes \mathcal{S}_{p}
$$

which leads to the resolution of the following system of linear equations:

$$
\sum_{\alpha \in \mathcal{J}_{m, p}} E\left(\mathbf{A} H_{\beta} H_{\alpha}\right) \mathbf{u}_{\alpha}=E\left(\mathbf{b} H_{\beta}\right), \quad \forall \beta \in \mathcal{J}_{m, p},
$$

where $\mathbf{u}_{\alpha}=\left(u_{1, \alpha} \ldots u_{n, \alpha}\right)^{T}$ and

$$
\begin{aligned}
& (\mathbf{A}(\boldsymbol{\xi}))_{i j}=\int_{D} \boldsymbol{\varepsilon}\left(\boldsymbol{\varphi}_{j}(\boldsymbol{x})\right): \boldsymbol{C}: \boldsymbol{\varepsilon}\left(\boldsymbol{\varphi}_{i}(\boldsymbol{x})\right) \mathcal{H}(-\phi(\boldsymbol{x}, \boldsymbol{\xi})) d \boldsymbol{x} \\
& (\mathbf{b})_{i}=\int_{\Gamma_{2}} \boldsymbol{\varphi}_{i} \cdot \boldsymbol{F} d s
\end{aligned}
$$

Properties of the approximate solution and computational aspects are detailed in [6].

\subsection{Numerical example}

We consider a plate with a hole of random shape defined in section 6.2. The plate lies in a random domain included in a fictitious domain $D=(0,1) \times(0,1)$. In this section, the aim is not to validate the identification procedure but to illustrate the efficiency of the $\mathrm{X}$ SFEM method, when the random level-set is given. For simplicity, we consider that the basic random variables $\boldsymbol{\xi}=\left(\xi_{1}, \ldots, \xi_{m}\right)$ are equal to random variables $\mathbf{Y}=\left(Y_{1}, \ldots, Y_{m}\right)$ appearing in equation (23). $\boldsymbol{\xi}=\left(\xi_{1}, \ldots, \xi_{5}\right)$ then denotes a vector of 5 independent uniform random variables $U(-\sqrt{3}, \sqrt{3})$, thus defining a stochastic domain $\Xi=(-\sqrt{3}, \sqrt{3})^{5}$. The level-set function is exactly decomposed on a Legendre polynomial chaos of degree $p=1$ in dimension 5 . We have shown in section 6.2 that the identification procedure led to an accurate representation with this polynomial chaos decomposition.

A plane strain assumption is made and a homogeneous isotropic material is considered with 


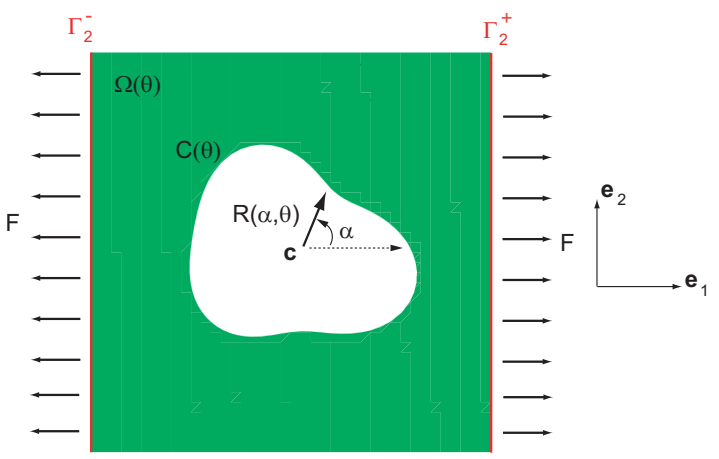

Figure 16. Plate with a hole in tension. Schematic representation of the rough boundary of the hole, defined in Eq. (22) and boundary conditions.

Young modulus equal to 1 and Poisson ratio equal to 0.3. The plate is subjected to a uniform unit tensile load $\boldsymbol{F}=( \pm 1,0)$ acting on the part $\Gamma_{2}=\Gamma_{2}^{-} \cup \Gamma_{2}^{+}$of its boundary. The problem is illustrated in Figure 16.

In the X-SFEM method, a unique mesh $\mathcal{T}_{h}$ is used, defined on the fictitious domain $D$ shown in Figure 17. The finite elements can be split into three groups: the first group $\left(e_{i}\right)$ comprises all elements being surely inside the domain, the second group $\left(e_{o}\right)$ contains all elements being surely outside the domain and the third group $\left(e_{c}\right)$ comprises all elements possibly cut by the boundary of the hole. A generalized polynomial chaos of order $p=3$ in dimension 5 is used for the approximation at the stochastic level. The basis functions of the approximation space $\mathcal{S}_{p}$ defined in section 7.2 are Legendre polynomials.

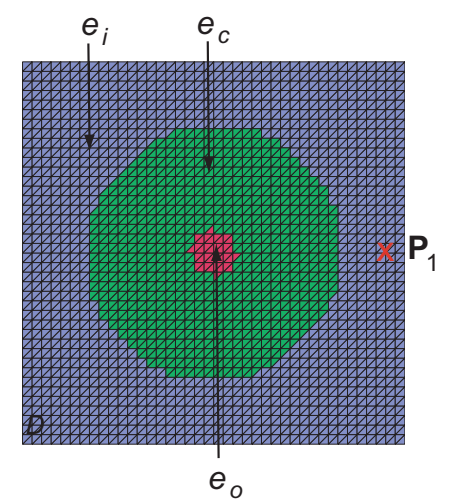

Figure 17. Random rough circle. X-SFEM mesh of fictitious domain $D$ with 3 groups of elements: surely in the domain $\left(e_{i}\right)$, surely outside the domain $\left(e_{o}\right)$ and possibly cut by the boundary $\left(e_{c}\right)$.

In order to illustrate the efficiency of the X-SFEM method, we compare various quantities obtained by post-processing of the X-SFEM solution with a reference solution obtained using a deterministic X-FEM approach. The X-SFEM solution being explicit in terms of basic random variables, post-processing can be performed at a very low cost. We first examine the horizontal 


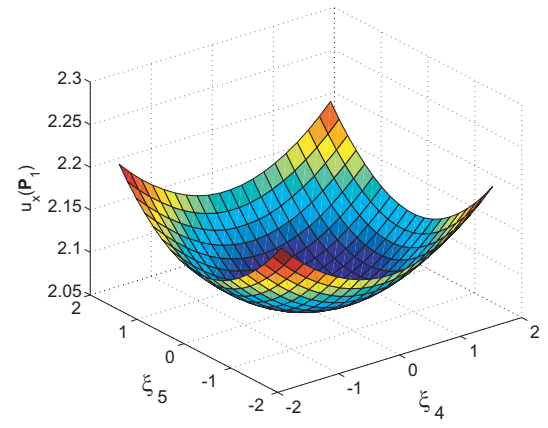

(a) X-FEM

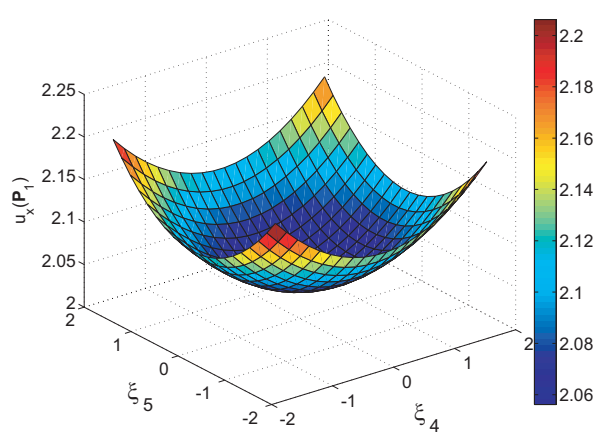

(b) X-SFEM with $p=3$

Figure 18. Random rough circle. Response surfaces for horizontal displacement at point $P_{1}$ as a function of $\xi_{4}$ and $\xi_{5}\left(\xi_{1}=\xi_{2}=\xi_{3}=0\right)$ : comparison between (a) deterministic X-FEM and (b) X-SFEM.

displacement $u_{x}$ of a particular point $P_{1}=(0.95,0.5)$ surely inside the domain, shown in Figure 17. The corresponding response surfaces are displayed as a function of two random variables $\left(\xi_{4}, \xi_{5}\right)$ while $\xi_{1}, \xi_{2}$ and $\xi_{3}$ are fixed to their mean values, which are equal to zero. Figure 18 shows the response surfaces of $u_{x}$ at $P_{1}$ obtained with the two approaches. We can observe a very good agreement between the X-SFEM and the reference (X-FEM) solutions.

Figure 19 presents the stress field component $\sigma_{x x}$ for a particular outcome of the random domain corresponding to $\boldsymbol{\xi}=(1,-1,1,-1,1)$. As in the case of the displacement, we observe a very good agreement between the two approaches in the calculation of the stresses. This indicates that the X-SFEM solution is accurate.

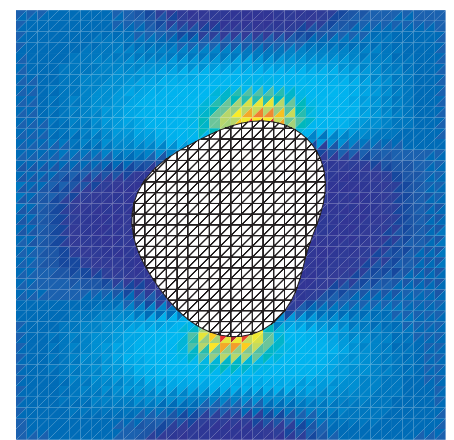

(a) deterministic X-FEM

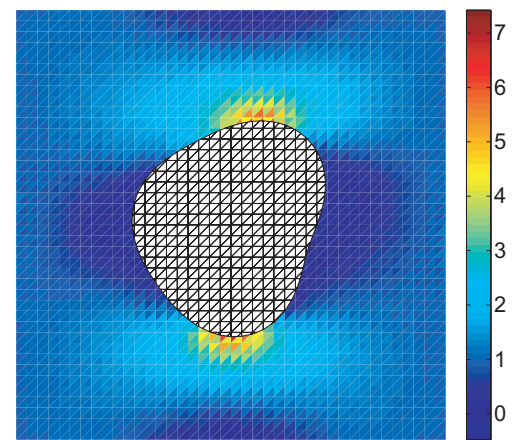

(b) X-SFEM with $\mathrm{p}=3$

Figure 19. Random rough circle. Stresses $\sigma_{x x}$ for one outcome of the random geometry $\Omega(\boldsymbol{\xi})$ with $\boldsymbol{\xi}=(1,-1,1,-1,1)$ : comparison between (a) deterministic X-FEM and (b) X-SFEM with $p=3$. 
We finally focus on the variability of the elastic strain energy $W(\boldsymbol{\xi})$ defined as

$$
W(\boldsymbol{\xi})=\frac{1}{2} \int_{\Omega(\boldsymbol{\xi})} \varepsilon(\boldsymbol{u}): \boldsymbol{C}: \boldsymbol{\varepsilon}(\boldsymbol{u}) d \boldsymbol{x}
$$

$W(\boldsymbol{\xi})$ has been approximated by its decomposition on a generalized Legendre polynomial chaos of order $p=3$. Knowing the X-SFEM solution $\boldsymbol{u}(\boldsymbol{x}, \boldsymbol{\xi}) \in \mathcal{V}_{n} \otimes \mathcal{S}_{p}$, the decomposition $W(\boldsymbol{\xi}) \approx \sum_{\alpha} W_{\alpha} H_{\alpha}(\boldsymbol{\xi})$ has been obtained by using an $L^{2}$-projection of $W(\boldsymbol{\xi})$ (use of Gaussian quadrature for computing the coefficients $\left.W_{\alpha}:=E\left(W(\boldsymbol{\xi}) H_{\alpha}(\boldsymbol{\xi})\right)\right)$. Figure 20 shows the response surface of $W(\boldsymbol{\xi})$ for X-FEM and X-SFEM respectively, with $\xi_{1}, \xi_{4}$ and $\xi_{5}$ fixed to their mean values and $\xi_{2}, \xi_{3}$ varying. In this figure, a good agreement between the two solutions can again be observed. The pdf of $W(\boldsymbol{\xi})$ obtained with X-SFEM and Monte Carlo simulation is finally examined in Figure 21. The Monte Carlo solution has been calculated using 10,000 samples and an X-FEM code to solve each deterministic problem. X-SFEM leads to a satisfactory matching of the pdf compared to that resulting from Monte Carlo simulation.

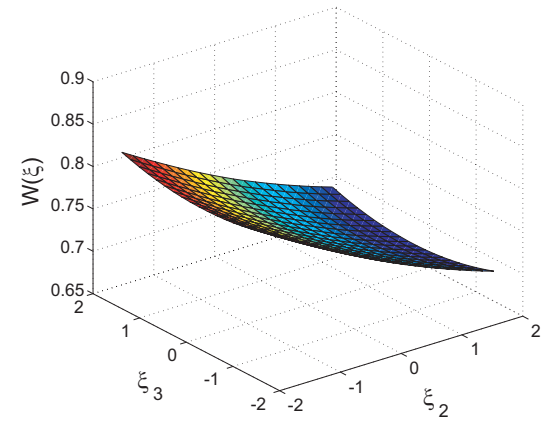

(a) X-FEM

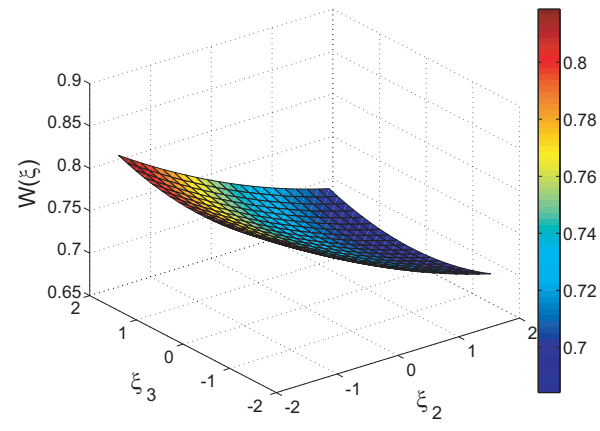

(b) X-SFEM with $p=3$

Figure 20. Random rough circle. Response surfaces for elastic strain energy $W(\boldsymbol{\xi})$ as a function of $\xi_{2}$ and $\xi_{3}\left(\xi_{1}=\xi_{4}=\xi_{5}=0\right)$ : comparison between (a) deterministic X-FEM and (b) X-SFEM.

\section{Conclusions}

In this paper, we have proposed an efficient identification procedure of random geometry in a form suitable for numerical simulation within the eXtended Stochastic Finite Element Method (X-SFEM). The method starts from a collection of images representing different outcomes of the random shape to identify. The key-point of the method is to represent the random geometry in an implicit manner using the level-set technique. In this context, the problem of random geometry identification is equivalent to the identification of a random level-set function, which is a random field. This random field is first decomposed using an empirical KarhunenLoève expansion, which allows to represent the samples of the level-set on a reduced basis of deterministic modes. The problem is thus transformed into the probabilistic identification of a few random variables, which are the components of the random level-set on this reduced basis of modes. The random variables are represented on a polynomial chaos basis and three efficient 


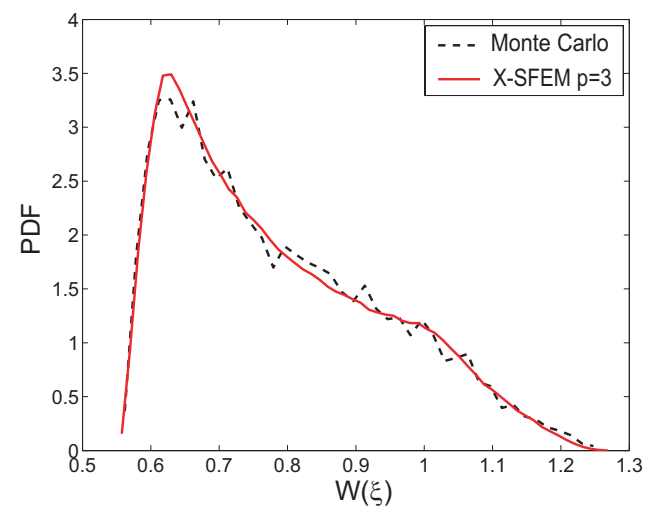

Figure 21. Random rough circle. Probability density function of $W(\boldsymbol{\xi})$ : comparison between X-SFEM and a Monte Carlo approach coupled with X-FEM (10 ${ }^{4}$ samples).

numerical strategies are used in order to identify the coefficients of their polynomial chaos decomposition: a maximum likelihood estimation without independence hypothesis $(\mathrm{ML}(\mathrm{D}))$, a maximum likelihood estimation with independence hypothesis $(\mathrm{ML}(\mathrm{I})$ ) and a projection method using empirical cumulative distribution functions (EP(I)). The performance of these strategies has been evaluated on some "manufactured" random geometry problems using various error criteria. It can be concluded that the two techniques assuming independence $(\mathrm{ML}(\mathrm{I})$ and $\mathrm{EP}(\mathrm{I}))$ are very competitive in terms of accuracy compared to the ML(D) approach, while the projection method $\mathrm{EP}(\mathrm{I})$ is the approach having the smallest computational cost for the identification procedure. This conclusion is in accordance with the assumption of independence which is very often used in practice due to the small collection of images that is usually available. Concerning the propagation of geometrical uncertainties in structural analysis, it can be stated that X-SFEM is capable of achieving a very good accuracy compared with a coupled (deterministic) X-FEM/Monte Carlo simulation approach.

\section{APPENDIX}

II. Parametrization of the Stiefel manifold $\mathbb{S}(n, m)$

We present here a possible parametrization of the Stiefel manifold $\mathbb{S}(n, m)$, with $m \leqslant n$, which is the set of $n$-by- $m$ orthogonal matrices. The dimension of the manifold is $d=n m-m(m+1) / 2$. In the following, we denote by $\mathbb{O}(n)$ the group of $n$-by- $n$ orthogonal matrices.

II.1. The general case $m \leqslant n$

A classical parametrization of $\mathbb{S}(n, m)$ around a matrix $\mathbf{B} \in \mathbb{S}(n, m)$ writes as follows:

$$
\mathbf{A}=\left(\mathbf{B} \mathbf{B}_{\perp}\right) \exp (\mathbf{S})\left(\begin{array}{c}
\mathbf{I}_{m} \\
0
\end{array}\right)
$$


where $\mathbf{B}_{\perp} \in \mathbb{S}(n, n-m)$ is the orthogonal complement of $\mathbf{B}$, i.e. $\left(\mathbf{B} \mathbf{B}_{\perp}\right) \in \mathbb{O}(n)$, and where $\mathbf{S} \in \mathbb{M}^{\text {skew }}(m)$ is a skew-symmetric matrix writing

$$
\begin{aligned}
& \mathbf{S}=\left(\begin{array}{cc}
\mathbf{S}_{11} & -\mathbf{S}_{21}^{T} \\
\mathbf{S}_{21} & 0
\end{array}\right), \\
& \mathbf{S}_{11} \in \mathbb{M}^{\text {skew }}(m), \mathbf{S}_{21} \in \mathbb{R}^{(n-m) \times m} .
\end{aligned}
$$

We can easily verify that the skew-symmetric matrices of the form (31) depend on $d$ independent parameters. Let $Z$ be the mapping defined on $\mathbb{R}^{d}$ such that $\mathbf{S}=Z(\varphi)$ is a matrix of the form (31), i.e.

$$
Z(\boldsymbol{\varphi})=\left(\begin{array}{ccc|cc}
0 & -\varphi_{1} & \ldots & -\varphi_{d^{\prime}+1} & \ldots \\
\varphi_{1} & \ddots & \ldots & \ldots & \ldots \\
\vdots & \vdots & 0 & \ldots & -\varphi_{d} \\
\hline \varphi_{d^{\prime}+1} & \vdots & \vdots & & 0 \\
\vdots & \vdots & \varphi_{d} & &
\end{array}\right)
$$

where the ordering of parameters has been arbitrarily chosen. The desired local parametrization of Stiefel manifold $\mathbb{S}(n, m)$ around matrix $\mathbf{B}$ can then be written:

$$
\mathbf{A}=T(\boldsymbol{\varphi})=\left(\mathbf{B} \mathbf{B}_{\perp}\right) \exp (Z(\boldsymbol{\varphi}))\left(\begin{array}{c}
\mathbf{I}_{m} \\
0
\end{array}\right)
$$

\section{II.2. The particular case $m=n$}

For $m<n, \mathbb{S}(n, m)$ is a connected manifold and can be completely spanned by the proposed parametrization. For $m=n, \mathbb{S}(n, m)=\mathbb{O}(n)$ is the group of orthogonal $n$-by- $n$ matrices, which is not a connected set. However, $\mathbb{O}(n)$ is the union of two connected sets $\mathbb{O}^{+}(n)$ and $\mathbb{O}^{-}(n)$, corresponding to the sets of orthogonal matrices respectively with determinant 1 and -1 . The proposed parametrization around a matrix $\mathbf{B}$ only allows to span the set to which $\mathbf{B}$ belongs, i.e. $\mathbb{O}^{+}(n)$ or $\mathbb{O}^{-}(n)$. Theoretically, an optimization problem on $\mathbb{O}(n)$ must then be separated into two optimization problems, using a parametrization around a matrix $\mathbf{B}$ respectively in $\mathbb{O}^{+}(n)$ and $\mathbb{O}^{-}(n)$.

For our purpose, the case $m=n$ appears for polynomial chaos expansions of degree 1 . In the case where we use a Hermite polynomial expansion of degree 1, we can easily show that the likelihood function $f(\mathbf{A})$ is invariant under all orthogonal transformations, i.e. $f(\mathbf{A})=f(\mathbf{O A})$, $\forall \mathbf{O} \in \mathbb{O}(n)$. This comes from the corresponding invariance of the probability density function of gaussian random vector $\boldsymbol{\xi}$. This means that $f$ is constant on $\mathbb{O}(n)$ and then, no optimization problem has to be solved. In the case where we use a Legendre chaos expansion of degree $1, f$ takes no more a constant value on $\mathbb{O}(n)$. However, we can show that optimization problems on $\mathbb{O}^{+}(n)$ and $\mathbb{O}^{-}(n)$ are completely equivalent. Indeed, for a matrix of coefficients $\mathbf{A} \in \mathbb{O}^{+}(n)\left(\right.$ resp. $\left.\mathbb{O}^{-}(n)\right)$, there exists $\mathbf{O} \in \mathbb{O}^{-}(n)$ such that $\mathbf{O A} \in \mathbb{O}^{-}(n)\left(\right.$ resp. $\left.\mathbb{O}^{+}(n)\right)$ and $f(\mathbf{A})=f(\mathbf{O A})$. This comes from invariance properties of the probability density function of random vector $\boldsymbol{\xi}$ with independent uniform random components. Then, only one optimization problem has to be solved on one of the connected sets of $\mathbb{O}(n)$. For other polynomial chaos basis, these invariance properties have to be analyzed in order to determine if one has to solve one or two optimization problems. 
However, we shall mention that chaos decompositions will generally be chosen with a degree greater than 2 and then, the case $m=n$ will not be encountered in practice.

\section{II.3. The particular case $m=1$}

In the case $m=1, \mathbb{S}(n, 1)$ is the unit hypersphere in $\mathbb{R}^{n}$. In the parametrization (31), this corresponds to $\mathbf{S}_{11}=0$ and $\mathbf{S}_{21}=\left(\varphi_{1}, \ldots, \varphi_{n-1}\right)^{T} \in \mathbb{R}^{n-1}$. With a little algebra, we can show that

$$
T(\boldsymbol{\varphi})=\left(\mathbf{B} \mathbf{B}_{\perp}\right)\left(\begin{array}{c}
\cos \left(\widetilde{\varphi}_{1}\right) \\
\sin \left(\widetilde{\varphi}_{1}\right) \varphi_{1} / \widetilde{\varphi}_{1} \\
\ldots \\
\sin \left(\widetilde{\varphi}_{1}\right) \varphi_{n-1} / \widetilde{\varphi}_{1}
\end{array}\right)
$$

By introducing the following change of variables:

$$
\begin{aligned}
\varphi_{1} & =\widetilde{\varphi}_{1} \cos \left(\widetilde{\varphi}_{2}\right) \\
\ldots & \\
\varphi_{n-2} & =\widetilde{\varphi}_{1} \sin \left(\widetilde{\varphi}_{2}\right) \ldots \sin \left(\widetilde{\varphi}_{n-2}\right) \cos \left(\widetilde{\varphi}_{n-1}\right) \\
\varphi_{n-1} & =\widetilde{\varphi}_{1} \sin \left(\widetilde{\varphi}_{2}\right) \ldots \sin \left(\widetilde{\varphi}_{n-2}\right) \sin \left(\widetilde{\varphi}_{n-1}\right),
\end{aligned}
$$

parametrization (33) is equivalent to

$$
T(\widetilde{\boldsymbol{\varphi}})=\left(\mathbf{B} \mathbf{B}_{\perp}\right)\left(\begin{array}{c}
\cos \left(\widetilde{\varphi}_{1}\right) \\
\sin \left(\widetilde{\varphi}_{1}\right) \cos \left(\widetilde{\varphi}_{2}\right) \\
\ldots \\
\sin \left(\widetilde{\varphi}_{1}\right) \ldots \sin \left(\widetilde{\varphi}_{n n-1}\right)
\end{array}\right)
$$

which is the classical parametrization of the hypersphere with $n-1$ angular parameters $\widetilde{\varphi} \in$ $\mathbb{R}^{n-1}$. We can notice that for parameters $\widetilde{\varphi}$, only a bounded domain $[0, \pi]^{n-2} \times[-\pi, \pi] \subset \mathbb{R}^{n-1}$ allows to completely span the hypersphere. This correspond to a bounded domain $[-\pi, \pi]^{n-1}$ for parameters $\varphi$. A bounded domain of parameters $[-\pi, \pi]^{d}$ also appears to be sufficient to completely span the Stiefel manifold $\mathbb{S}(n, m)$ in the general case $n<m$.

\section{REFERENCES}

1. Ghanem R, Spanos P. Stochastic finite elements: a spectral approach. Springer, Berlin, 1991.

2. Babuška I, Tempone R, Zouraris GE. Solving elliptic boundary value problems with uncertain coefficients by the finite element method: the stochastic formulation. Computer Methods in Applied Mechanics and Engineering 2005; 194:1251-1294.

3. Xiu DB, Tartakovsky DM. Numerical methods for differential equations in random domains. SIAM J. Sci. Comput. 2006; 28(3):1167-1185.

4. Canuto C, Kozubek T. A fictitious domain approach to the numerical solution of pdes in stochastic domains. Numerische Mathematik 2007; 107(2):257-293.

5. Nouy A, Schoefs F, Moës N. X-SFEM, a computational technique based on X-FEM to deal with random shapes. European Journal of Computational Mechanics 2007; 16(2):277-293.

6. Nouy A, Clément A, Schoefs F, Moës N. An extended stochastic finite element method for solving stochastic partial differential equations on random domains. Computer Methods in Applied Mechanics and Engineering 2008; 197:4663-4682.

7. Moës N, Dolbow J, Belytschko T. A finite element method for crack growth without remeshing. Int. J. for Numerical Methods in Engineering 1999; 46:131-150. 
8. Daux C, Moës N, Dolbow J, Sukumar N, Belytschko T. Arbitrary branched and intersecting cracks with the extended finite element method. Int. J. for Numerical Methods in Engineering 2000; 48:1741-1760.

9. Sukumar N, Chopp D, Moës N, Belytschko T. Modeling holes and inclusions by level sets in the extended finite-element method. Computer Methods in Applied Mechanics and Engineering 2001; 190:6183-6200.

10. Belytschko T, Moës N, Usui S, Parami C. Arbitrary discontinuities in finite elements. Int. J. for Numerical Methods in Engineering 2001; 50(4):993-1013.

11. Belytschko T, Parimi C, Moës N, Sukumar N, Usui S. Structured extended finite element methods for solids defined by implicit surfaces. Int. J. for Numerical Methods in Engineering 2003; 56:609-635.

12. Sethian J. Level Set Methods and Fast Marching Methods: Evolving Interfaces in Computational Geometry, Fluid Mechanics, Computer Vision, and Materials Science. Cambridge University Press, Cambridge, UK, 1999.

13. Desceliers C, Ghanem R, Soize C. Maximum likelihood estimation of stochastic chaos representations from experimental data. Int. J. for Numerical Methods in Engineering 2006; 66(6):978-1001.

14. Ghanem R, Doostan A. On the construction and analysis of stochastic models: characterization and propagation of the errors associated with limited data. Journal of Computational Physics 2006; 217(1):6381 .

15. Mitchell IM. The flexible, extensible and efficient toolbox of level set methods. Journal of Scientific Computing 2007; 25(2-3):300-329.

16. Loève M. Probability Theory. II, fourth edition, in: Graduate Texts in Mathematics, vol. 46. SpringerVerlag, New York, 1978.

17. Jolliffe I. Principal Component Analysis. 2nd edn., Springer, New York, 2002.

18. Fukunaga K. Introduction to Statistical Pattern Recognition. Elsevier, 1990.

19. Silverman BW. Smoothed functional principal components analysis by choice of norm. Ann. Statist. 1996; 24(1):1-24.

20. Levy A, Rubinstein J. Some properties of smoothed principal component analysis for functional data. Journal of The Optical Society of America 1999; 16(1):28-35.

21. Kirby M. Minimal dynamical systems from pdes using sobolev eigenfunctions. Physica D: Nonlinear Phenomena 1992; 57(3-4):466-475.

22. Berkooz G, Holmes P, Lumley JL. The proper orthogonal decomposition in the analysis of turbulent flows. Annual review of fluid mechanics $1993 ; \mathbf{2 5}: 539-575$.

23. Wiener N. The homogeneous chaos. Am. J. Math. 1938; 60:897-936.

24. Soize C, Ghanem R. Physical systems with random uncertainties: chaos representations with arbitrary probability measure. SIAM J. Sci. Comput. 2004; 26(2):395-410.

25. Xiu DB, Karniadakis GE. The Wiener-Askey polynomial chaos for stochastic differential equations. SIAM J. Sci. Comput. 2002; 24(2):619-644.

26. Gentle J. Elements of Computational Statistics. Springer, New York, 2002.

27. Edelman A, Arias TA, Smith ST. The geometry of algorithms with orthogonality constraints. SIAM J. Matrix Anal. Appl. 1998; 20(2):303-353.

28. Fang KT, Li RZ. Some methods for generating both an nt-net and the uniform distribution on a stiefel manifold and their applications. Computational Statistics \& Data Analysis 1997; 24:29-46. 